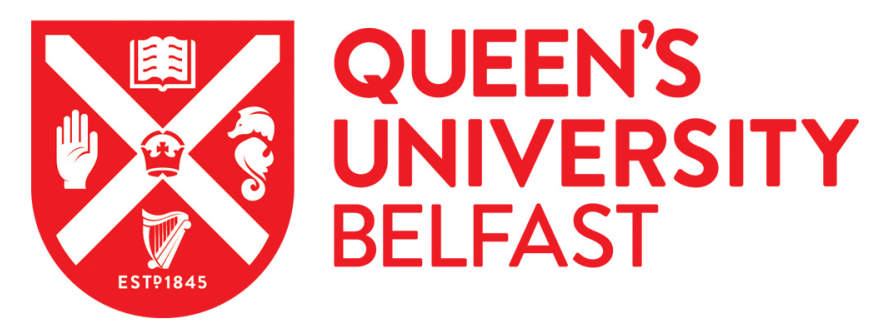

\title{
Wireless Powered Wearables using Distributed Massive MIMO
}

Dinh Van, S., Ngo, H-Q., \& Cotton, S. L. (2020). Wireless Powered Wearables using Distributed Massive MIMO. IEEE Transactions on Communications. https://doi.org/10.1109/TCOMM.2020.2965442

\author{
Published in: \\ IEEE Transactions on Communications
}

\section{Document Version:}

Peer reviewed version

Queen's University Belfast - Research Portal:

Link to publication record in Queen's University Belfast Research Portal

Publisher rights

Copyright 2020 IEEE. This work is made available online in accordance with the publisher's policies. Please refer to any applicable terms of use of the publisher.

\section{General rights}

Copyright for the publications made accessible via the Queen's University Belfast Research Portal is retained by the author(s) and / or other copyright owners and it is a condition of accessing these publications that users recognise and abide by the legal requirements associated with these rights.

Take down policy

The Research Portal is Queen's institutional repository that provides access to Queen's research output. Every effort has been made to ensure that content in the Research Portal does not infringe any person's rights, or applicable UK laws. If you discover content in the Research Portal that you believe breaches copyright or violates any law, please contact openaccess@qub.ac.uk. 


\title{
Wireless Powered Wearables using Distributed Massive MIMO
}

\author{
Son Dinh Van, Student Member, IEEE, Hien Quoc Ngo, Member, IEEE and Simon L. Cotton, Senior \\ Member, IEEE
}

\begin{abstract}
This paper presents an analytical framework which lays the foundation for distributed massive multiple-input multiple-output (MIMO) supported wireless power transfer for wearable devices. In our approach, we consider multiple users, each wearing a number of wireless sensors along with one body worn hub which acts as a relay to forward information from the on-body sensors to nearby access points (APs). Each AP is equipped with a large number of antennas and is not only responsible for receiving data sent from the hubs, but also supplying wireless power to them. Interaction between hubs and APs is not exclusive to a single pairing, with APs assigned to supply energy to and receive data from more than one hub. More precisely, APs perform the downlink energy transmission and the uplink data transmission using maximum-ratio combining. Analytical approximations of the outage probability and spectral efficiency are derived. Based on these analytical results, two modes of operation are investigated. These are outage probability prioritized and spectral efficiency prioritized. For each mode, maxmin power controls are proposed to ensure a uniformly good service throughout the area of coverage. By contrasting with the IEEE 802.15.6 standard, the numerical results illustrate that while using collocated massive MIMO may provide unsatisfactory performance, the distributed setting shows much promise for enabling wireless power transfer for wearable devices.
\end{abstract}

Index Terms-Distributed Massive MIMO, Outage Probability Analysis, Rician Fading, Wearable Networks.

\section{INTRODUCTION}

Recent years have witnessed a massive growth in the number of wireless devices designed to be worn on the human body. This is in part due to significant advances in computing, hardware design and communication innovations meaning that wearable technology has been encroaching on various aspects of our life [1]. For example, more and more wearable devices are being designed to interact with our bodies for health monitoring purposes, collecting vital signs such as heart rate and blood pressure [2], [3]. Despite these improvements, battery technology has not advanced at the same rate. As a consequence, tremendous effort has been put into finding new materials and innovative methods of charging so as to deliver a better user experience [4]. One such approach is through the use of wireless power transfer, which enables devices to harvest energy from ambient electromagnetic radiations in the surrounding environment [5]. Energy harvesting wireless

S. Dinh, H. Q. Ngo and S. L. Cotton are with the Centre for Wireless Innovation, Institute of Electronics, Communications and Information Technology, The Queen's University of Belfast, Queen's Road, Belfast, BT3 9DT, UK Email: \{sdinh01, hien.ngo, simon.cotton\}@qub.ac.uk. The work of H. Q. Ngo was supported by the UK Research and Innovation Future Leaders Fellowships under Grant MR/S017666/1. networks are expected to introduce a number of benefits including energy-sufficiency, perpetual operation and an ability to deploy wireless networks in difficult-to-reach places such as within the human body.

Two energy harvesting mechanisms referred to as time switching or power splitting, were introduced in [6]. It was demonstrated that time switching outperforms power splitting in the case of high transmission rates, low signal-to-noise-ratio (SNR) regimes, or low energy harvesting efficiency. In [7], the authors proposed protocols for wireless-powered relay nodes in cooperative communications with energy accumulation at the relay based on the time switching method. Despite these contributions, it is worth highlighting that two significant concerns still remain for the implementation of wireless power transfer. Firstly, only a small portion of the energy radiated by a wireless power source can typically be harvested. Secondly, wireless power transfer suffers heavily from path loss, shadowing and multipath fading. As a result, if a receiver is located too far from a power source, the total wireless energy it is capable of accumulating may be insufficient to fulfil its needs. This will be especially pertinent for systems operating with only one antenna at both the energy harvesting devices and power source such as in [6], [7].

Massive multiple-input multiple-output (MIMO), where the base station (BS) is equipped with a large number of antennas, may pave the way for wireless power transfer owing to its ability to beam energy towards a specific spatial area. Massive antenna arrays can be utilized in either collocated or distributed architectures. In collocated massive MIMO systems, all antennas are located in a compact area. By contrast, in distributed massive MIMO systems, all the antennas are spread out over a large area to guarantee that almost anywhere in the region may experience a line-of-sight (LOS) with at least one antenna [8]. It is anticipated that the distributed massive MIMO system may benefit wireless power transfer owing to its ability to take advantage of macro diversity to overcome shadow fading.

The idea of utilizing collocated massive MIMO to enable wireless power transfer has been of interest in a number of studies [9]-[11]. In [9], wireless information and power transfer in large-scale MIMO systems was considered. A resource allocation scheme was introduced by jointly optimizing the energy harvesting duration and the transmit power while considering a delay constraint. The authors in [10] investigated the overall power transfer efficiency and the energy efficiency of a wireless powered massive MIMO system where the BS is capable of charging single-antenna users. Subsequently, these 
users utilize the harvested energy to transmit information to the BS. The research proposed in [12] focused on utilizing wireless power transfer enabled massive MIMO with imperfect CSI. In particular, the minimum rate among users were maximized by jointly optimizing the allocation of time and energy resources. Despite providing some useful insights, all of the above studies restricted their analyses and performance evaluations of wireless powered networks to Rayleigh fading channels, which is often not realistic in practice. The research conducted in [11] investigated the feasibility of using massive antenna arrays to enable wireless power transfer for wireless sensor nodes over Rician fading channels. It was shown that a significant difference exists in the performance of wireless powered networks when the associated channels used for power transfer are in LOS or non-LOS states.

While all of the above research (with the exception of [11]) concentrated on investigating wireless power transfer to prolong the life time of user equipment in cellular networks, there has been a limited number of studies which investigate this technology for wearable networks. One of the most important performance metrics used to evaluate the performance of a wearable network is outage probability. Considering body area networks (BANs) as an example, the IEEE 802.15.6 standard suggests that the achieved outage probability should be lower than $10 \%$. Unfortunately, analyzing outage probability in the context of massive MIMO is quite challenging. Despite this, there have been a few studies which have investigated this problem. For example, in [13], the authors proposed an approximation for the outage probability in a massive MIMO downlink system when treating the interference as a random variable. The result showed that the outage probability increases as the number of users becomes larger. In [14], an exact expression for the outage probability of any user in a single-cell multiple-user MIMO network operating over independent Rayleigh fading channels was proposed. The results indicated that if the number of antennas at the BS is very large, the outage probability exhibits a deterministic behavior. However, the outage probability approximations introduced in [13], [14] are complicated and cannot readily be applied for further analyses. It is important to note that massive MIMO has been utilized successfully to reduce power consumption in the domain of wireless sensor networks. In [15]-[17], the authors showed that the power consumed by the sensors decays proportionally to the number of antennas at the fusion center.

In this paper, we consider wireless power transfer to wearable devices using a distributed massive MIMO system, where a large number of service antennas, called access points (APs), serve a number of users distributed over a nearby area. Each user wears a number of sensors to collect biological data and a hub node which acts as a relay to transfer data from the sensors to the APs. All APs cooperate via a backhaul network, and serve all users in the same time-frequency resource via time-division duplex (TDD) operation. Each hub is allowed to choose a number of APs to request wireless energy from and communicate with. Conjugate beamforming is used by each AP for both off-body downlink energy (i.e. AP-to-wearable device) and uplink data (i.e. wearable deviceto-AP) transmission. The main contributions of our work may now be summarized as follows. Firstly, unlike previous works which utilize only one AP, in this paper, we consider an advanced system model in which there is more than one AP and multiple users. In addition, the small-scale fading of the wireless channel between each hub and AP is modeled using the Rician fading model where the power delivered by the LOS component varies depending on the relative distance of the link. As a result, the models introduced in [9]-[11] are a special case of our system model. Our second contribution is the proposal of an approximation for the outage probability ${ }^{1}$ of the received signal at a central processing unit (CPU). To obtain this, the inter-user interference is approximated using the moment-matched method and the law of large numbers. Not only is the proposed approximation shown to be more tractable than previous methods introduced in the literature such as [13], [14], it can also be easily tailored for use in further analyses. Numerical results are also provided to validate the accuracy of the approximation. Finally, two transmission power strategies are proposed for all APs to ensure a uniform quality of service for all users. In particular, one strategy is aimed at wearable applications where there is a focus on achieving a low outage probability as the highest priority (so-called outage probability prioritized algorithm), whereas the other scheme targets high-data rate applications which prioritize data rate more than outage (i.e. a spectral efficiency prioritized strategy). Numerical results are provided and then contrasted with the IEEE 802.15.6 standard to validate the performance of these algorithms.

Notation: Throughout this paper, we use lowercase and uppercase boldface letters to represent vectors and matrices, respectively. The transpose, conjugate-transpose and inverse of $\mathbf{X}$ are denoted by $\mathbf{X}^{T}, \mathbf{X}^{H}$ and $\mathbf{X}^{-1}$. The notation $\mathbf{X} \succ 0$ indicates that all entries of $\mathbf{X}$ are positive while $\operatorname{vec}($.$) is a$ vectorization operator. In addition, $\mathbf{I}_{M}$ stands for an $M \times M$ identity matrix; $X_{i j}$ and $\mathbf{X}[n]$ give the $(i, j)$-th entry and the $n$-th row of $\mathbf{X}$ whereas $\mathbf{X} \sim \mathcal{C N}(\mathbf{M}, \mathbf{V})$ denotes that $\mathbf{X}$ is a complex Gaussian matrix with mean matrix $\mathbf{M}$ and covariance matrix V. Finally, $\mathbb{E}\{$.$\} and \mathbb{V}\{$.$\} represent the expectation$ and variance operator, $\|$.$\| is the Euclidean norm while \Re($. and $\Im($.$) denote the real and the imaginary operator.$

\section{SYSTEM MODEL}

In this paper, we consider a wireless network consisting of $N$ hybrid data-and-energy APs serving $K$ users, as illustrated in Figure 1. Each AP is equipped with $M$ antennas and all $N$ APs are distributed around a specific deployment area to supply wireless energy for and receive data from $K$ users (or equivalently wearable devices). Additionally, all APs connect to a CPU via a backhaul network. We assume that all $N$ APs simultaneously serve all users in the same time-frequency resource. Each user avails of a number of biological sensors along with one hub node which acts as a relay to transfer the collected data from the sensors to the APs. As the hub nodes act as both a central sink and forwarding devices on the body, we consider utilizing multiple APs to simultaneously supply

\footnotetext{
${ }^{1}$ In this paper, we define outage probability as the probability that the received SINR is lower than a pre-defined SINR threshold.
} 


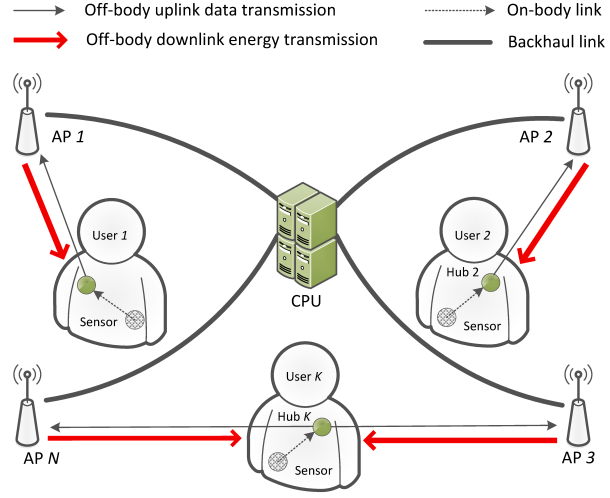

Fig. 1. The system model.

RF energy to power the hubs. As a consequence, in our model, all the hubs are allowed to select $N_{0}$ out of $N$ APs to harvest energy from their RF signals $\left(1 \leq N_{0} \leq N\right)$.

In this paper, we denote APs and hubs by their indexes (e.g. AP $n$ stands for the $n$-th AP and hub $k$ is the hub positioned on the $k$-th user). Moreover, we denote $\mathcal{A}_{k}$ as the group of APs serving hub $k$, while $\mathcal{H}_{n}$ stands for the group of hubs served by AP $n$. Note that $\mathcal{A}_{k}$ contains $N_{0}$ index elements and $\mathcal{H}_{n}$ can be an empty set.

\section{A. Channel Model}

In this work, the complex channel vector between AP $n$ and hub $k$ is modeled as [18]

$$
\mathbf{h}_{n k}=\sqrt{\beta_{n k}}\left(\sqrt{\frac{\mathcal{K}_{n k}}{\mathcal{K}_{n k}+1}} \mathbf{a}_{n k}+\sqrt{\frac{1}{\mathcal{K}_{n k}+1}} \mathbf{z}_{n k}\right),
$$

where $\mathcal{K}_{n k}$ is the Rician $\mathcal{K}$-factor, $\beta_{n k}$ represents the largescale fading coefficient. In addition, $\mathbf{a}_{n k}$ and $\mathbf{z}_{n k}$ correspond to the LOS and non-LOS (NLOS) components, respectively. It is assumed that $\mathbf{z}_{n k} \sim \mathcal{C N}\left(\mathbf{0}_{M}, \mathbf{I}_{M}\right), \mathbf{a}_{n k}$ is a vector satisfying $\left\|\mathbf{a}_{n k}\right\|^{2}=M$. Moreover, we consider low mobility scenarios, and hence, $\mathbf{a}_{n k}, \mathcal{K}_{n k}$ and $\beta_{n k}$ change very slowly with respect to time and can be assumed to be deterministic for the signal processing duration of interest [19]. These terms can be easily estimated by following the method proposed in [20].

For the sake of tractability, we denote $d_{n k} \triangleq \frac{\beta_{n k}}{\mathcal{K}_{n k}+1}, \overline{\mathbf{h}}_{n k} \triangleq$ $\sqrt{\frac{\beta_{n k} \mathcal{K}_{n k}}{\mathcal{K}_{n k}+1}} \mathbf{a}_{n k}$ and $\widetilde{\mathbf{h}}_{n k} \triangleq \sqrt{d_{n k}} \mathbf{z}_{n k}$. As a result, $\mathbf{h}_{n k}$ can be rewritten as $\mathbf{h}_{n k}=\overline{\mathbf{h}}_{n k}+\widetilde{\mathbf{h}}_{n k}$. Obviously, $\overline{\mathbf{h}}_{n k}$ is a deterministic vector and $\widetilde{\mathbf{h}}_{n k} \sim \mathcal{C N}\left(\mathbf{0}_{M}, d_{n k} \mathbf{I}_{M}\right)$; hence, $\mathbf{h}_{n k} \sim \mathcal{C N}\left(\overline{\mathbf{h}}_{n k}, d_{n k} \mathbf{I}_{M}\right)$. In addition, $\left\|\overline{\mathbf{h}}_{n k}\right\|^{2}=M \mathcal{K}_{n k} d_{n k}$.

\section{B. Transmission Protocol}

We assume that all the APs, sensors and hubs are perfectly synchronized and operate with a TDD protocol. In addition, that each AP has up-to-date and accurate knowledge of the local channel state information (CSI) of the links to all of the

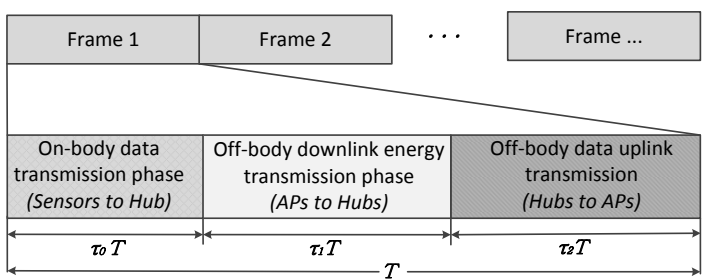

Fig. 2. An illustration of one frame structure for the proposed system model.

hubs it serves. ${ }^{2}$ We also assume that the channel coherence time is on the order of, or greater than the frame length. In this paper, frame-based transmission is considered. As shown in Figure 2, the length of each frame is fixed, lasts for $T$ seconds and consists of three phases. More precisely:

- First phase - On-body data transmission: All sensors send their collected data to its corresponding on-body hub node in a fixed time period of $\tau_{0} T$ seconds, with $0<\tau_{0}<1$. For each user, there is one hub which is responsible for collecting all the data for the next phase. Note that all data transmitted by the sensors is forwarded to the hub node with no acknowledgement. As we are focusing on the transfer of energy to and data from the hub nodes, this phase is not explicitly considered in the analysis of our system model.

- Second phase - Off-body downlink energy transmission: During this phase of length $\tau_{1} T$ seconds $\left(0<\tau_{1}<1\right)$, each hub chooses $N_{0}$ out of $N$ APs to request energy. Subsequently, these APs deliver wireless energy to the corresponding hubs via beamforming.

- Third phase - Off-body uplink data transmission: During the remaining time of $\tau_{2} T$ seconds $\left(0<\tau_{2}<1\right)$, each hub simultaneously reports its collected information to the APs it selected in the second phase. Each hub will utilize the total energy harvested in the second phase for this transmission. Subsequently, all of the APs process the received information before forwarding it to the CPU via backhaul links.

Note that it is a necessary condition for $\tau_{0}+\tau_{1}+\tau_{2}=1$. For convenience, without loss of generality, we normalize $T=1$.

\footnotetext{
${ }^{2}$ In practice, each AP can estimate the channels to all of the hubs via the uplink training phase. This is done once for each coherence interval. The details of channel estimation process follow those given in [21]. As a result, the normalized mean-square error (NMSE) of the channel estimation corresponding to channel $\mathbf{h}_{n k}$ is NMSE $\mathrm{N}_{n k}=\frac{1}{\tau_{p} p_{p} \beta_{n k}+\mathcal{K}_{n k}+1}$, where $\tau_{p}$ is the training duration for each coherence interval, and $p_{p}$ is the normalized pilot power. We can see that if $\tau_{p}$ is long enough, then $\mathrm{NMSE}_{n k}$ is very close to 0 which implies the channel estimate is very close to the true channel. Since $\tau_{p}$ is limited by the coherence interval $\tau_{c}$, we can chose large $\tau_{p}$ only when $\tau_{c}$ is very large. Fortunately, in our work, we consider wearable applications. For wearable applications, the coherence interval is very large (thousands of symbols or more). According to [22], the coherence time measured for wearable applications operatung in an indoor environment is $380.8 \mathrm{~ms}, 125.1 \mathrm{~ms}$ and $27.07 \mathrm{~ms}$ for standing, walking and jogging, respectively. In [23], the authors concluded that a coherence time of $25 \mathrm{~ms}$ is a reasonable assumption for most scenarios. With a coherence time of $25 \mathrm{~ms}$, and a coherence bandwidth of $1 \mathrm{MHz}$, the coherence interval is equal to the (coherence time) $\times($ coherence bandwidth) which is 25000 symbols. Thus we can use a large duration for uplink training. For example, if we choose $\tau_{p}=1000$ symbols to obtain very small NMSE, the corresponding channel estimation overhead will be just $1000 / 25000=0.04$ which is negligible. Therefore, the assumption of perfect CSI in this application is reasonable.
} 
1) Off-body Downlink Energy Transmission Phase: In this phase, all the APs simultaneously beam wireless energy toward the corresponding hubs. Each AP utilizes a weighted sum of conjugate beamformers, also known as maximum-ratio transmission, for each hub in the downlink. We choose this beamforming technique since it was shown that this is the optimal beamformer that maximizes the harvested energy in the case when $M$ is large [12]. Taking AP $n$ as an example, the signal generated can be formulated as

$$
\mathbf{s}_{n}=\sum_{i \in \mathcal{H}_{n}} \sqrt{\xi_{n, i} P_{\mathrm{m}}} \frac{\mathbf{h}_{n i}}{\left\|\mathbf{h}_{n i}\right\|} x_{n i},
$$

where $\xi_{n, i}$ is the weighted power corresponding to hub $i$ with $i \in \mathcal{H}_{n}$ and $\boldsymbol{\xi}_{n}$ is the vector of weights utilized by AP $n$. These weights are subject to the conditions: $\xi_{n, i}>0$ and $\sum_{i \in \mathcal{H}_{n}} \xi_{n, i} \leq 1$. In addition, $P_{\mathrm{m}}$ stands for the maximum transmit power of AP $n$ (it is assumed that all APs have an identical maximum transmit power), while $x_{n i}$ represents the symbol transmitted to hub $i$ from AP $n$ with $i \in \mathcal{H}_{n}$. Note that $x_{n i}$ is the data symbol which is independent of other symbols such as $\mathbb{E}\left\{x_{n i}\right\}=0, \mathbb{E}\left\{\left\|x_{n i}\right\|^{2}\right\}=1$. For tractability, we denote $\xi \triangleq\left[\begin{array}{llll}\boldsymbol{\xi}_{1}^{T} & \boldsymbol{\xi}_{2}^{T} & \cdots & \boldsymbol{\xi}_{N}^{T}\end{array}\right]^{T}$. Then the signal received by hub $k$ can be written as

$$
y_{k}=\sum_{n=1}^{N} \mathbf{h}_{n k}^{H} \mathbf{s}_{n}+n_{k},
$$

where $n_{k}$ is the additive noise at hub $k, n_{k} \sim \mathcal{C N}\left(0, \sigma_{n}^{2}\right)$ with $\sigma_{n}^{2}$ standing for the noise power.

It is assumed that all the hubs have infinite battery storage to store the harvested energy. Note that only a fraction of the energy emitted from the APs is harvested by each hub. In this paper, we adopt the linear energy conversion model ${ }^{3}$. Let $\delta$ be the energy conversion efficiency $(0<\delta<1)$, the total amount of energy harvested by hub $k$ is given by

$$
E_{k}\left(\tau_{1}, \boldsymbol{\xi}\right)=\delta \tau_{1} \mathbb{E}\left\{\left|y_{k}\right|^{2}\right\} .
$$

This term will be derived in detail in Section III.

2) Off-body Uplink Data Transmission Phase: In the uplink data transmission phase, all the hubs simultaneously send the collected data to the corresponding APs in a time duration of $\tau_{2} T$ seconds using the RF energy harvested in the second phase. Therefore, the transmit power, $p_{k}$, utilized by hub $k$ can is

$$
p_{k}=p_{k}\left(\tau_{1}, \tau_{2}, \boldsymbol{\xi}\right)=\frac{E_{k}\left(\tau_{1}, \boldsymbol{\xi}\right)}{\tau_{2}} .
$$

Remark 1. In reality, $p_{k}$ is limited by the maximum transmit power. In the majority of cases, the value of $p_{k}$ which is used is much lower than this limit due to the amount of energy which can be realistically harvested from energy transmissions in phase 2 .

Let's consider an arbitrary hub, i.e. hub $k$, which sends its data to group $\mathcal{A}_{k}$. Then each $\mathrm{AP}$ in group $\mathcal{A}_{k}$ (i.e. for

\footnotetext{
${ }^{3}$ Recently, the non-linear model for modeling the wireless energy harvested by a transceiver has been considered [24]. Nonetheless, the linear model is still often favored thanks to its tractability and its ability to act as the first order approximation of the non-linear model [12], [25].
}

$\forall n \in \mathcal{A}_{k}$ ) will receive a $M \times 1$ received signal vector, which is given by

$$
\mathbf{y}_{n}=\mathbf{h}_{n k} x_{k}+\sum_{i=1, i \neq k}^{K} \mathbf{h}_{n i} x_{i}+\mathbf{n}_{n}, \text { with } \forall n \in \mathcal{A}_{k},
$$

where $\mathbf{n}_{n} \sim \mathcal{C N}\left(\mathbf{0}_{M}, \sigma_{n}^{2} \mathbf{I}_{M}\right)$ is the noise vector while $x_{i}$ stands for the signal transmitted by hub $i$ with $x_{i}=\sqrt{p_{i}} s_{i}$. Here, $s_{i}$ is the data symbol which is independent of symbols generated from other hubs.

In order to decode the signal transmitted from hub $k$, each AP in the group $\mathcal{A}_{k}$ utilizes a maximal-ratio combining (MRC) receiver ${ }^{4}$. As such, the received signal after the decoder stage at $\mathrm{AP} n \in \mathcal{A}_{k}$ can be written as

$$
t_{k}(n)=\left\|\mathbf{h}_{n k}\right\|^{2} x_{k}+\sum_{i=1, i \neq k}^{K} \mathbf{h}_{n k}^{H} \mathbf{h}_{n i} x_{i}+\mathbf{h}_{n k}^{H} \mathbf{n}_{n} .
$$

After decoding the signal, all the APs in group $\mathcal{A}_{k}$ simultaneously forward the decoded signal to the CPU via a backhaul link for the final detection. Therefore, the received signal for hub $k$ at the CPU is

$$
t_{k}=\sum_{n \in \mathcal{A}_{k}}\left\|\mathbf{h}_{n k}\right\|^{2} x_{k}+\sum_{\substack{i=1 \\ i \neq k}}^{K} \sum_{n \in \mathcal{A}_{k}} \mathbf{h}_{n k}^{H} \mathbf{h}_{n i} x_{i}+\sum_{n \in \mathcal{A}_{k}} \mathbf{h}_{n k}^{H} \mathbf{n}_{n} .
$$

In (8), the first term is considered as the desired signal transmitted from hub $k$, while the second term is regarded as the inter-user interference which is the total interference coming from the other $K-1$ hubs.

To reduce the complexity of (8), with $\forall k, i \in 1, \cdots, K$, we define $\mathbf{l}_{k i}$ as the vectorization of $\mathbf{h}_{n i}$ (with $\forall n \in \mathcal{A}_{k}$ ), which is formulated by stacking a $\mathbf{h}_{n i}$ vector (with $\forall n \in \mathcal{A}_{k}$ ) on top of another. In particular, this term can be expressed as $\mathbf{l}_{k i} \triangleq$ $\operatorname{vec}\left(\mathbf{h}_{n i}, \forall n \in \mathcal{A}_{k}\right)=\left[\mathbf{h}_{n i}^{T} \mathbf{h}_{m i}^{T} \ldots\right]^{T}$ with $\forall n, m \in \mathcal{A}_{k}$. As a result, $\mathbf{l}_{k i} \sim \mathcal{C N}\left(\overline{\mathbf{l}}_{k i}, \mathbf{L}_{k i}\right)$ where $\mathbf{1}_{k i}$ and $\mathbf{L}_{k i}$ are the mean vector and the covariance matrix, respectively. It is not difficult to show that $\overline{\mathbf{l}}_{k i}=\operatorname{vec}\left(\overline{\mathbf{h}}_{n i}, \forall n \in \mathcal{A}_{k}\right)$, whereas the covariance matrix $\mathbf{L}_{k i} \triangleq \operatorname{diag}(\underbrace{d_{n i}, \cdots, d_{n i}}_{M \text { elements }}, \underbrace{d_{m i}, \cdots, d_{m i}}_{M \text { elements }}, \cdots)$, with $\forall n, m \in \mathcal{A}_{k}$. It is worth noting that $\overline{\mathbf{l}}_{k i}$ and $\mathbf{L}_{k i}$ are deterministic owing to the fact that the mean and variance of all channel vectors are deterministic. Interestingly, we have the following relationships

$$
\sum_{n \in \mathcal{A}_{k}} \mathbf{h}_{n k}^{H} \mathbf{h}_{n i}=\mathbf{l}_{k k}^{H} \mathbf{l}_{k i}, \quad \sum_{n \in \mathcal{A}_{k}}\left\|\mathbf{h}_{n k}\right\|^{2}=\left\|\mathbf{l}_{k k}\right\|^{2} .
$$

As a result, the received signal for hub $k$ at the CPU, which is derived in (8) now can be reduced to

$$
t_{k}=\left\|\mathbf{l}_{k k}\right\|^{2} x_{k}+\sum_{i=1, i \neq k}^{K} \mathbf{l}_{k k}^{H} \mathbf{l}_{k i} x_{i}+\mathbf{l}_{k k} \mathbf{n}_{k},
$$

\footnotetext{
${ }^{4}$ The MRC decoder performs very well when the number of antennas is large. More importantly, it is very simple, has low computational complexity, and can be implemented in a distributed manner [26]. Other advanced linear processing techniques such as zero-forcing may enhance the system performance. However, these methods typically require a significant volume of information sent to the CPU, which in turn, may put significant burden on the backhaul network [21].
} 
where $\mathbf{n}_{k}$ is the combined noise vector. This general derivation is useful for determining the performance metrics which will be introduced in the next section.

Remark 2. In some circumstances, the signal received at the CPU for the stream generated by hub $k$ (so-called the stream $k$ ) contains very strong interference plus noise relative to the desired signal. This phenomenon occurs when hub $k$ is positioned very far from the APs in group $\mathcal{A}_{k}$, while other hubs (in the same group) stay close to this group. In these circumstances, the power weights designed for hub $k$ should be sufficiently great to compensate for the inter-user interference. In Section IV, we will introduce algorithms which aim to overcome this challenge.

\section{PERformance AnAlysis}

In this section, we derive closed-form expressions of the signal-to-interference-plus-noise-ratio (SINR), achievable rate, and outage probability which enable us to obtain important insights as well as design the power control and AP allocation for supplying wireless power to wearable devices within our system.

\section{A. Mathematical Preliminaries}

This section presents some useful results which will be used later to analyze the performance metrics of our system model.

Lemma 1. The expectation of the norm square of the inner product between any two channel vectors is

$$
\begin{aligned}
& g_{n}[k, i] \triangleq \mathbb{E}\left\{\left|\mathbf{h}_{n k}^{H} \mathbf{h}_{n i}\right|^{2}\right\} \\
& = \begin{cases}\beta_{n k}^{2}\left(M^{2}+\frac{4 M \mathcal{K}_{n k}+2 M}{\left(\mathcal{K}_{n k}+1\right)^{2}}\right), & \text { if } i=k . \\
\frac{\beta_{n k} \beta_{n i}\left(M+M \mathcal{K}_{n i}+M \mathcal{K}_{n k}+\mathcal{K}_{n i} \mathcal{K}_{n k}\left|\mathbf{a}_{n k}^{H} \mathbf{a}_{n i}\right|^{2}\right)}{\left(\mathcal{K}_{n k}+1\right)\left(\mathcal{K}_{n i}+1\right)}, & \text { if } i \neq k .\end{cases}
\end{aligned}
$$

Proof. See [27].

Lemma 2. The expectation of the inner product of two identical combined channel vectors is

$$
\mathbb{E}\left\{\left\|\mathbf{l}_{k k}\right\|^{2}\right\}=M \sum_{n \in \mathcal{A}_{k}} \beta_{n k} \triangleq r_{k} .
$$

Proof. See Appendix VII-A.

Lemma 3. The expectation of the norm-square of the inner product of two combined channel vectors is

$$
\begin{aligned}
& q_{k i} \triangleq \mathbb{E}\left\{\left|\mathbf{l}_{k k}^{H} \mathbf{l}_{k i}\right|^{2}\right\} \\
& = \begin{cases}\sum_{n \in \mathcal{A}_{k}} \mathbf{g}_{n}[k, k]+\sum_{n \neq m \in \mathcal{A}_{k}} M^{2} \beta_{n k} \beta_{m k}, & \text { if } i=k . \\
\sum_{n \in \mathcal{A}_{k}} \mathbf{g}_{n}[k, i]+\sum_{n \neq m \in \mathcal{A}_{k}} \overline{\mathbf{h}}_{n i}^{H} \overline{\mathbf{h}}_{n k} \overline{\mathbf{h}}_{m k}^{H} \overline{\mathbf{h}}_{m i}, \text { if } i \neq k .\end{cases}
\end{aligned}
$$

Proof. See Appendix VII-B.

Lemma 4. The asymptotic inner product of two identical combined channel vectors is given by

$$
\frac{1}{M}\left\|\mathbf{l}_{k k}\right\|^{2} \stackrel{\text { a.s. }}{\rightarrow} \sum_{n \in \mathcal{A}_{k}} \beta_{n k}, \text { as } M \rightarrow \infty,
$$

where $\stackrel{\text { a.s. }}{\rightarrow}$ denotes the almost sure convergence.

Proof. See Appendix VII-C.

Lemma 5. The asymptotic norm square of the inner product between two identical combined channel vectors is expressed as

$$
\frac{1}{M^{2}}\left\|\mathbf{l}_{k k}\right\|^{4} \stackrel{\text { a.s. }}{\rightarrow}\left(\sum_{n \in \mathcal{A}_{k}} \beta_{n k}\right)^{2} \text {, as } M \rightarrow \infty .
$$

Proof. This can be obtained directly by using Lemma 4 and [28, Theorem 1].

\section{B. SINR Analysis}

From (10), the instantaneous SINR of stream $k$ can be expressed as

$$
\gamma_{k}\left(\tau_{1}, \tau_{2}, \boldsymbol{\xi}\right)=\frac{p_{k}\left\|\mathbf{l}_{k k}\right\|^{4}}{\sum_{i=1, i \neq k}^{K} p_{i}\left|\mathbf{l}_{k k}^{H} \mathbf{l}_{k i}\right|^{2}+\left\|\mathbf{l}_{k k}\right\|^{2}} .
$$

In general, analyzing performance metrics related to the SINR can be very challenging since $X_{k}$ contains the combinations of many random variables. To reduce the complexity of the analysis, it is necessary to obtain an approximation for the transmit power $p_{k}$, which is presented in the following lemma. The approximation is obtained from the asymptotic results in previous section.

Lemma 6. With the beamformer in (2), the transmit power utilized by Hub $k$ can be approximated by $\tilde{p}_{k}$ by

$$
\tilde{p}_{k} \triangleq \delta \frac{\tau_{1}}{\tau_{2}}\left(\sum_{n \in \mathcal{A}_{k}} M \beta_{n k} P_{m} \xi_{n, k}+\sum_{\substack{n=1 \\ i \in \mathcal{H}_{n}}}^{N} \sum_{\substack{i \neq k \\ i \in \mathcal{H}_{m}}} P_{m} \frac{g_{n}[k, i]}{M \beta_{n i}}\right)
$$

with $k=1, \cdots, K$.

Proof. See Appendix VII-D.

The formula (17) can be rewritten as:

$$
\tilde{p}_{k}=\frac{\tau_{1}}{\tau_{2}} \sum_{n=1}^{N} \sum_{i \in \mathcal{A}_{n}} \varrho_{k}[n, i] \xi_{n, i},
$$

where $\varrho_{k}[n, i]$ stands for the sum of the terms associated to $\xi_{n, i}$. The first term in (17) is the harvested energy from the beam directed toward hub $k$ from the APs in the group $\mathcal{H}_{k}$, while the second term represents the total energy collected from beams directed toward other hubs from all APs but still harvested by hub $k$. For the sake of tractability, we denote $\mathbf{p} \triangleq\left[\tilde{p}_{1}, \tilde{p}_{2}, \cdots, \tilde{p}_{K}\right]^{T}$. In addition, let $\mathbf{G}$ be the matrix whose the $k$-th row is

$$
\mathbf{G}[k]=\left[\varrho_{k}[1]^{T} \varrho_{k}[2]^{T} \cdots \varrho_{k}[N]^{T}\right]^{T},
$$

where $\varrho_{k}[n]$ is a column vector whose the $i$-th element is $\varrho_{k}[n, i]$ with $i \in \mathcal{A}_{n}$. From (17), now we have

$$
\mathbf{p}=\frac{\tau_{1}}{\tau_{2}} \mathbf{G} \boldsymbol{\xi} .
$$


Proposition 1. When $M$ is sufficiently large, the SINR of the stream $k$ can be approximated to

$$
\tilde{\gamma}_{k}\left(\tau_{1}, \tau_{2}, \boldsymbol{\xi}\right) \triangleq \frac{\tilde{p}_{k} r_{k}^{2}}{\sum_{i=1, i \neq k}^{K} \tilde{p}_{i}\left|\mathbf{l}_{k k}^{H} \mathbf{l}_{k i}\right|^{2}+r_{k}} .
$$

and the expectation of SINR of the stream $k$ can be approximated to

$$
\mathbb{E}\left\{\gamma_{k}\left(\tau_{1}, \tau_{2}, \boldsymbol{\xi}\right)\right\} \approx \frac{\tilde{p}_{k} q_{k k}}{\sum_{i=1, i \neq k}^{K} \tilde{p}_{i} q_{k i}+r_{k}} \triangleq \bar{\gamma}_{k} .
$$

\section{Proof. See Appendix VII-E.}

\section{Spectral Efficiency Analysis}

The spectral efficiency of the uplink data transmission from hub $k$ is formulated as

$$
\mathcal{R}_{k}=\mathcal{R}_{k}\left(\tau_{1}, \tau_{2}, \boldsymbol{\xi}\right) \triangleq \tau_{2} \mathbb{E}\left\{\log _{2}\left[1+\gamma_{k}\left(\tau_{1}, \tau_{2}, \boldsymbol{\xi}\right)\right]\right\} .
$$

In general, the formula given in (23) is relatively complicated to compute. Hence, a tight approximation for $\mathcal{R}_{k}$ will be introduced in the following lemma.

Proposition 2. The spectral efficiency of the uplink data transmission from hub $k$ can be approximated to

$$
\tilde{\mathcal{R}}_{k} \triangleq \tau_{2} \log _{2}\left(1+\frac{\tilde{p}_{k} q_{k k}}{\sum_{i=1, i \neq k}^{K} \tilde{p}_{i} q_{k i}+r_{k}}\right) .
$$

Proof. See Appendix VII-F.

\section{Outage Probability Analysis}

In this section, a novel approximation for the outage probability is derived. We define outage probability as a probability that the received SINR falls below a pre-defined SINR threshold, $\gamma_{t h}$. In particular, the outage probability of the received signal for hub $k$ is formulated as

$$
\mathcal{P}_{k} \triangleq \mathcal{P}_{k}\left(\tau_{1}, \tau_{2}, \boldsymbol{\xi}\right) \triangleq \mathbb{P}\left[\gamma_{k}\left(\tau_{1}, \tau_{2}, \boldsymbol{\xi}\right)<\gamma_{t h}\right] .
$$

In general, obtaining a closed-form derivation for $\mathcal{P}_{k}$ is very challenging since it contains various non-identical random variables. However, by using Proposition 1, the outage probability of stream $k$ can be approximated to

$$
\tilde{\mathcal{P}}_{k} \triangleq \mathbb{P}\left[\tilde{\gamma}_{k}\left(\tau_{1}, \tau_{2}, \boldsymbol{\xi}\right)<\gamma_{t h}\right]=\mathbb{P}\left[\frac{\tilde{p}_{k} r_{k}^{2}}{X_{k}+r_{k}}<\gamma_{t h}\right],
$$

where $X_{k}$ stands for the inter-user interference term, i.e. $X_{k} \triangleq$

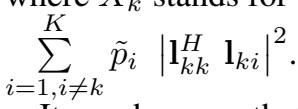

It can be seen that when $M$ is sufficiently large, the terms representing the desired signal and noise can be treated as deterministic, whereas the inter-user interference term $X_{k}$ can be considered as a RV. Unfortunately, obtaining a closedform solution for the cumulative distribution function (CDF) of $X_{k}$ in elementary functions appears to be infeasible due to $X_{k}$ containing $K-1$ non-identical random variables.
To circumvent with this limitation, the moment matching approximation is used. This method was shown to be effective in obtaining the approximation to the sum distribution [29]. In particular, we approximate $X_{k}$ by another random variable $\tilde{X}_{k}$ whose the first and second moments are identical to that of $X_{k}$, where $\tilde{X}_{k}$ follows a Gamma distribution, i.e. $\tilde{X}_{k} \sim \Gamma\left(A_{k}, B_{k}\right)$ with $A_{k}, B_{k}$ standing for the shape and the rate parameter. This means that $\mathbb{E}\left[\tilde{X}_{k}\right]=\mathbb{E}\left[X_{k}\right]$ and $\mathbb{V}\left[\tilde{X}_{k}\right]=\mathbb{V}\left[X_{k}\right]$, which are equivalent to

$$
\frac{A_{k}}{B_{k}}=\mathbb{E}\left\{X_{k}\right\}, \quad \frac{A_{k}}{B_{k}^{2}}=\mathbb{V}\left\{X_{k}\right\} .
$$

Thus, the shape $A_{k}$ and rate $B_{k}$ of the approximated Gamma distributed RV $\tilde{X}_{k}$ can be determined as

$$
A_{k}=\frac{\left(\mathbb{E}\left\{X_{k}\right\}\right)^{2}}{\mathbb{V}\left\{X_{k}\right\}}, \quad B_{k}=\frac{\mathbb{E}\left\{X_{k}\right\}}{\mathbb{V}\left\{X_{k}\right\}} .
$$

To obtain the approximation, it is necessary to compute the expectation and the variance of $X_{k}$.

Computation of the expectation: The expectation of $X_{k}$ can be obtained by using Lemma 3.

$$
\mathbb{E}\left\{X_{k}\right\}=\sum_{i=1, i \neq k}^{K} \tilde{p}_{i} q_{k i} .
$$

Computation of the variance: The variance of $X_{k}$ can be determined from $\mathbb{V}\left\{X_{k}\right\}=\mathbb{E}\left\{X_{k}^{2}\right\}-\left(\mathbb{E}\left\{X_{k}\right\}\right)^{2}$. The expectation of $X_{k}^{2}$ is expressed as

$$
\begin{aligned}
& \mathbb{E}\left\{X_{k}^{2}\right\}=\sum_{i=1, i \neq k}^{K} \tilde{p}_{i}^{2} \underbrace{\mathbb{E}\left\{\left|\begin{array}{ll}
\mathbf{l}_{k k}^{H} & \mathbf{l}_{k i}
\end{array}\right|^{4}\right\}}_{\text {term } 1}
\end{aligned}
$$

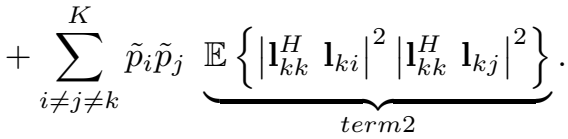

Now, two lemmas are introduced to obtain expressions for term 1 and term 2 .

Lemma 7. Let $y_{m}$ and $y_{v}$ be the mean and the variance of $\mathbf{l}_{k k}^{H} \mathbf{l}_{k i}$, respectively. Then, $y_{m}$ and $y_{v}$ can be expressed as

$$
y_{m} \triangleq \overline{\mathbf{l}}_{k k}^{H} \overline{\mathbf{l}}_{k i}, \quad y_{v} \triangleq q_{k i}-\left|\overline{\mathbf{l}}_{k k}^{H} \overline{\mathbf{l}}_{k i}\right|^{2} .
$$

In addition, when $M$ is sufficiently large, term 1 can be approximated to

$$
\operatorname{term} 1 \approx\left|y_{m}\right|^{4}+4 y_{v}\left|y_{m}\right|^{2}+2 y_{v}^{2}
$$

Proof. See Appendix VII-G.

Lemma 8. The closed-form expression for term 2 is represented as

$$
\begin{aligned}
\operatorname{term} 2 & =2 \operatorname{Tr}\left(\mathbf{B L}_{k k} \mathbf{C} \mathbf{L}_{k k}\right)+4 \overline{\mathbf{l}}_{k k}^{H} \mathbf{B} \mathbf{L}_{k k} \mathbf{C} \overline{\mathbf{l}}_{k k}+\left(\operatorname{Tr}\left(\mathbf{L}_{k k} \mathbf{B}\right)\right. \\
& \left.+\overline{\mathbf{l}}_{k k}^{H} \mathbf{B} \overline{\mathbf{l}}_{k k}\right)\left(\operatorname{Tr}\left(\mathbf{L}_{k k} \mathbf{C}\right)+\overline{\mathbf{l}}_{k k}^{H} \mathbf{C} \overline{\mathbf{l}}_{k k}\right),
\end{aligned}
$$

where $\mathbf{B} \triangleq \mathbf{L}_{k i}+\overline{\mathbf{l}}_{k i} \overline{\mathbf{l}}_{k i}^{H}$ and $\mathbf{C} \triangleq \mathbf{L}_{k j}+\overline{\mathbf{l}}_{k j} \overline{\mathbf{l}}_{k j}^{H}$.

Proof. See Appendix VII-H.

By using Lemma 7 and 8 , the expectation of $X_{k}^{2}$ is obtained. Subsequently, the parameters $A_{k}, B_{k}$ for the approximated 


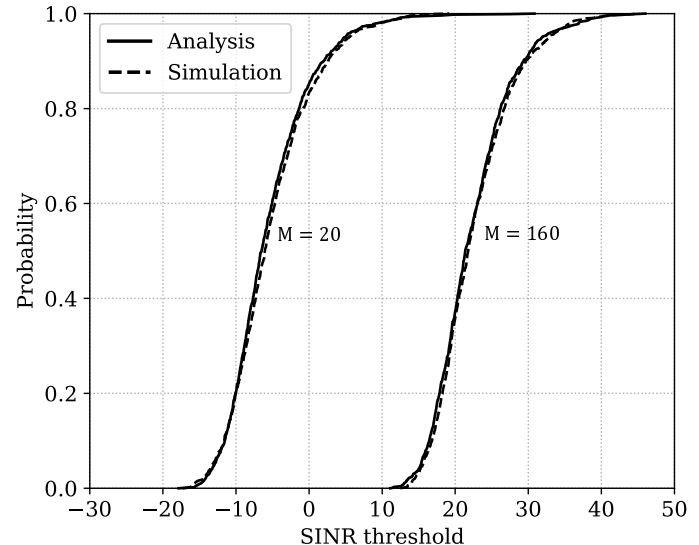

Fig. 3. The CDF of the received SINR for hub 0 in 2 cases: $M=20$ and $M=160$. In this simulation, random power weights were utilized at each AP, while other parameters used are $N=9, K=10, N_{0}=2, \tau_{1}=$ $0.8, \tau_{2}=0.2$.

Gamma distribution can be acquired by substituting (29) and (30) into (28). Based on this, the outage probability of stream $k$ can be approximated as

$$
\begin{aligned}
\tilde{\mathcal{P}}_{k} & \approx \mathbb{P}\left[\frac{\tilde{p}_{k} r_{k}^{2}}{\tilde{X}_{k}+r_{k}}<\gamma_{t h}\right]=\mathbb{P}\left[\tilde{X}_{k}>\frac{\tilde{p}_{k}}{\gamma_{t h}} r_{k}^{2}-r_{k}\right] \\
& = \begin{cases}1, & \text { if } \frac{\gamma_{t h}}{\tilde{p}_{k}} \geq r_{k} \\
1-\Gamma\left[A_{k}, B_{k}\left(\frac{\tilde{p}_{k}}{\gamma_{t h}} r_{k}^{2}-r_{k}\right)\right], & \text { otherwise }\end{cases}
\end{aligned}
$$

where $\Gamma(a, x)$ is the incomplete Gamma function defined as $\Gamma(a, x)=\frac{1}{\Gamma(a)} \int_{0}^{x} t^{a-1} e^{-t} d t$. In addition, $\Gamma(\cdot)$ is Gamma function [30].

The most complicated computation is encountered when obtaining term 2 from Lemma 8. However, this term is a deterministic number and can be calculated effectively. In contrast, the final approximation of the outage probability appears in a simple form which contains only one special function. Compared to other approximations introduced in the literature such as [13], [14], the proposed method is shown to be more tractable and particularly suited for application to other analyses. Although this approximation has been designed for use in this paper, it can readily be applied to other system models in the context of massive MIMO. To validate the accuracy of the proposed approximation, Figure 3 compares the outage probability achieved by hub 0 using analytical computation (with (33)) and simulation. In this comparison, the locations of the hubs and APs were fixed and there were 1000 small-scale fading realizations generated for the simulation. In addition, random power weights were utilized at each AP. As can be seen, the obtained analytical result matches the simulated data perfectly for the both cases: low $M(M=20)$ and large $M(M=160)$.

\section{POWER CONTROL AND AP Allocation}

Power control is an important concept, which has been used in massive MIMO for many years. For example, in [31], the authors investigated optimal joint pilot and data power allocation in single cell uplink massive MIMO systems to optimize the spectral efficiency. It is also well known that wireless power transfer is susceptible to propagation phenomenon such as path loss and shadowing, which may cause unfair resource allocation issues among users. In this section we present some power control methods to ensure fairness among users and discuss how the APs are assigned to users.

\section{A. Max-min Power Control Mechanisms}

In our system model, there are two modes of operation, which are:

- Outage probability prioritized mode (OP mode): An example of which is low-rate BAN devices, which typically consist of sensors which sample with low frequency. As a result, outage probability is considered as the key priority.

- Spectral efficiency prioritized mode (SEP mode): Examples which use SEP include high-rate BANs, which place an emphasis on guaranteeing a high data rate as opposed to a particular outage probability.

In this section, we introduce power control mechanisms for each mode. The power control algorithms are performed at the CPU.

1) Optimization Problem Formulated for OP Mode: The optimization problem designed for obtaining the fairness among users in terms of outage probability is formulated as

$$
\begin{aligned}
& \min _{\tau_{1}, \tau_{2}, \boldsymbol{\xi}} \max _{k=1, \ldots, K} \mathcal{P}_{k}\left(\tau_{1}, \tau_{2}, \boldsymbol{\xi}\right) \\
& \text { s.t } \tau_{0}+\tau_{1}+\tau_{2}=1 \\
& \sum_{i \in \mathcal{H}_{n}} \xi_{n, i} \leq 1 \quad, \forall n=1, \cdots, N \\
& \mathcal{R}_{k}\left(\tau_{1}, \tau_{2}, \boldsymbol{\xi}\right) \geq \mathcal{R}_{t h} \quad, \forall k=1, \cdots, K \\
& \tau_{1}, \tau_{2}>0, \boldsymbol{\xi} \succ 0,
\end{aligned}
$$

where (34b) is due to the frame duration constraint, and (34c) is due to the power constraint at each AP, while (34d) is to guarantee that the spectral efficiency of each hub is higher than a pre-defined spectral efficiency threshold $\mathcal{R}_{t h}$ and (34e) means that all the time durations and power weights are greater than 0 .

In general, solving this optimization problem is challenging since the objective function (34a) contains an incomplete Gamma function. Therefore, we focus on solving a suboptimal optimization problem in the case when $M$ is large, which is represented as

$$
\begin{aligned}
\max _{\tau_{1}, \tau_{2}, \boldsymbol{\xi}} & \min _{k=1, \ldots, K} \bar{\gamma}_{k}\left(\tau_{1}, \tau_{2}, \boldsymbol{\xi}\right) \\
\text { s.t } & (34 b),(34 c),(34 e) \\
& \tilde{\mathcal{R}}_{k}\left(\tau_{1}, \tau_{2}, \boldsymbol{\xi}\right) \geq \mathcal{R}_{t h}, \quad \forall k=1, \cdots, K .
\end{aligned}
$$

It is important to note that the solution obtained from solving (35) can be optimal or suboptimal.

2) Optimization Problem Formulated for SEP Mode: To achieve fairness among users in terms of the spectral efficiency, we investigate the following optimization problem

$$
\begin{array}{cl}
\max _{\tau_{1}, \tau_{2}, \boldsymbol{\xi}} \min _{k=1, \ldots, K} \tilde{R}_{k}\left(\tau_{1}, \tau_{2}, \boldsymbol{\xi}\right) \\
\text { subject to } & (34 b),(34 c),(34 e) \\
& \tilde{\mathcal{P}}_{k}\left(\tau_{1}, \tau_{2}, \boldsymbol{\xi}\right) \leq \mathcal{P}_{t h}, \quad \forall k=1, \cdots, K,
\end{array}
$$




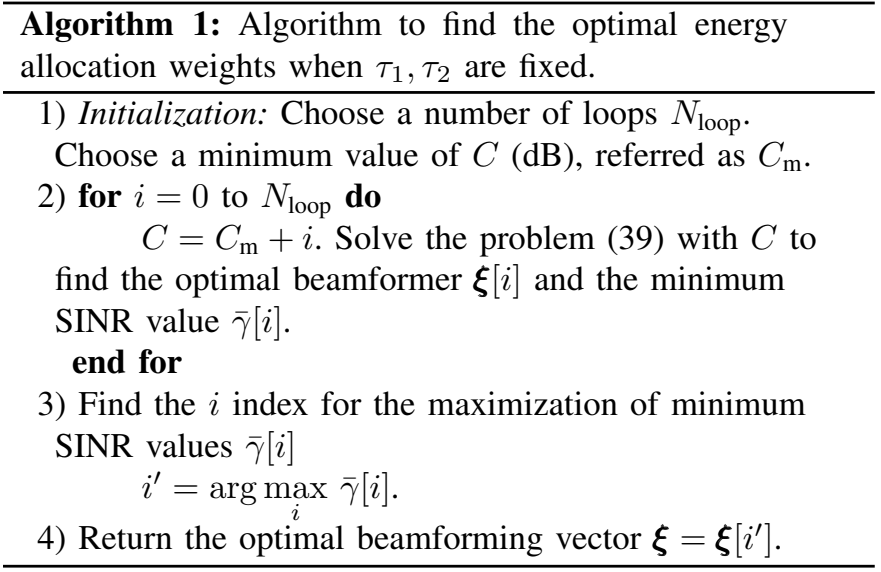

where (36d) is to guarantee that the outage probability of each uplink stream is lower than a pre-defined outage threshold $\mathcal{P}_{t h}$.

\section{B. Solving the Optimization Problem Designed for OP Mode}

1) Optimal Power Allocation Weights: In this subsection, we fix $\tau_{1}, \tau_{2}$, and investigate the optimal energy allocation weights for the optimization problem (35).

The minimum average SINR is maximized when all hubs achieve the same average SINR [12]. Let's define $\bar{\gamma}_{1}=\bar{\gamma}_{2}=$ $\cdots=\bar{\gamma}_{k}=C$, which is equivalent to

$$
\begin{aligned}
& \frac{\tilde{p}_{k} q_{k k}}{\sum_{i=1, i \neq k}^{K} \tilde{p}_{i} q_{k i}+r_{k}}=C, \forall k=1, \cdots, K \\
& \Leftrightarrow \tilde{p}_{k} q_{k k}-\sum_{i=1, i \neq k}^{K} C \tilde{p}_{i} q_{k i}=C r_{k}, \forall k=1, \cdots, K .
\end{aligned}
$$

By treating $C$ as a constant and defining $\mathbf{q}, \mathbf{Q}$ as

$$
\begin{aligned}
& \mathbf{q} \triangleq C \times\left[r_{1}, r_{2}, \cdots, r_{K}\right]^{T}, \\
& \mathbf{Q} \triangleq\left[\begin{array}{ccccc}
q_{11} & -C q_{12} & -C q_{13} & \cdots & -C q_{1 K} \\
-C q_{21} & q_{22} & -C q_{23} & \cdots & -C q_{2 K} \\
\cdots & & & & \\
-C q_{K 1} & -C q_{K 2} & -C q_{K 3} & \cdots & q_{K K}
\end{array}\right],
\end{aligned}
$$

the set of equations represented in (37) can be rewritten as $\mathbf{Q p}=\mathbf{q}$. Since $\mathbf{p}=\frac{\tau_{1}}{\tau_{2}} \mathbf{G} \boldsymbol{\xi}$, we obtain $\frac{\tau_{1}}{\tau_{2}} \mathbf{Q G} \boldsymbol{\xi}=\mathbf{q}$. Let $\overline{\mathbf{G}} \triangleq \frac{\tau_{1}}{\tau_{2}} \mathbf{Q G}$, the optimization problem for solving $\boldsymbol{\xi}$ in the case when $\tau_{1}, \tau_{2}$ are fixed now is equivalent to

$$
\begin{aligned}
\min _{\boldsymbol{\xi}} & \|\overline{\mathbf{G}} \boldsymbol{\xi}-\mathbf{q}\|^{2} \\
\text { subject to } & \sum_{i \in \mathcal{H}_{n}} \xi_{n, i} \leq 1, \quad \forall n=1, \cdots, N \\
& \boldsymbol{\xi} \succ 0 .
\end{aligned}
$$

It can be seen that (39) is a least squares optimization problem with inequality constraints, which can be effectively solved using convex optimization software packages such as cvxpy [32]. The algorithm used to solve $\boldsymbol{\xi}$ in the case when $\tau_{1}$ and $\tau_{2}$ are fixed is presented in Algorithm 1.

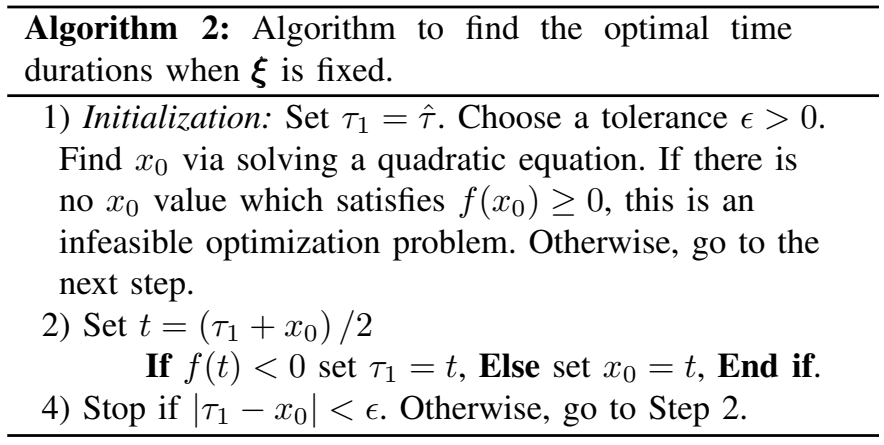

2) Optimal Time Durations: In this subsection, we fix $\xi$ and aim at investigating the optimal time durations ( $\tau_{1}$ and $\tau_{2}$ ) for the optimization problem (35).

Defining $k^{\prime} \triangleq \arg \min _{k} \bar{\gamma}_{k}$, the objective function $\min \bar{\gamma}_{k}$ can be rewritten as

$$
\begin{aligned}
\bar{\gamma}_{k^{\prime}}\left(\tau_{1}, \tau_{2}\right) & =\frac{\frac{\tau_{1}}{\tau_{2}} \mathbf{G}\left[k^{\prime}\right] \boldsymbol{\xi} q_{k^{\prime} k^{\prime}}}{\sum_{\substack{i=1, i \neq k_{1}^{\prime}}}^{\tau_{1}} \mathbf{G}\left[k^{\prime}\right] \boldsymbol{\xi} q_{k^{\prime} i}+r_{k^{\prime}}} \\
& =\frac{\lambda}{\mu+\frac{\tau_{2}}{\tau_{1}} \nu}=\frac{\lambda \tau_{1}}{\mu \tau_{1}+\nu \tau_{2}},
\end{aligned}
$$

where $\lambda, \mu$ and $\nu$ are all constants defined as $\lambda \triangleq \mathbf{G}\left[k^{\prime}\right] \xi q_{k^{\prime} k^{\prime}}$, $\mu \triangleq \sum_{i=1, i \neq k^{\prime}} \mathbf{G}\left[k^{\prime}\right] \xi q_{k^{\prime} i}$ and $\nu \triangleq r_{k^{\prime}}$.

Since $\tau_{1}+\tau_{2}=1-\tau_{0} \triangleq \hat{\tau}$, maximizing $\gamma_{k^{\prime}}\left(\tau_{1}, \tau_{2}\right)$ is equivalent to maximizing $\tau_{1}$. As a result, we now have an equivalent optimization problem as follows

$$
\begin{array}{ll}
\max _{\tau_{1}} & \tau_{1} \\
\text { s.t } & \log _{2}\left(1+\frac{\lambda \tau_{1}}{\mu \tau_{1}+\nu\left(\hat{\tau}-\tau_{1}\right)}\right)-\frac{\mathcal{R}_{t h}}{\hat{\tau}-\tau_{1}} \geq 0 \\
& 0<\tau_{1}<\hat{\tau},
\end{array}
$$

where (41b) is constructed from (35d). Interestingly, this optimization problem turns into case we wish to find the maximum answer of the equation $f(x)=0$ in the range $(0, \hat{\tau})$, where

$$
f(x)=\log _{2}\left(1+\frac{\lambda x}{\mu x+\nu(\hat{\tau}-x)}\right)-\frac{\mathcal{R}_{t h}}{\hat{\tau}-x} .
$$

Note that the $\lim _{x \rightarrow 0} f(x)=-\frac{\mathcal{R}_{t h}}{\tau_{1}}<0$ and the $\lim _{x \rightarrow \hat{\tau}} f(x)=$ $-\infty<0$. Let's define $x_{0}$ as the local optimal point satisfying $f\left(x_{0}\right)=\max _{0<x<\hat{\tau}} f(x) . x_{0}$ can be found via solving $f^{\prime}\left(x_{0}\right)=0$, which is equivalent to solving the following quadratic equation

$$
a x^{2}+b x+c=0,
$$

where $a \triangleq \mathcal{R}_{t h}(\mu-\nu)(\lambda+\mu-\nu)-\lambda \nu \hat{\tau} / \log (2) ; b \triangleq$ $\nu \hat{\tau}(\lambda+2 \mu-2 \nu) \mathcal{R}_{t h}+2 \lambda \nu \hat{\tau}^{2} / \log (2)$ and $c \triangleq \nu^{2} \hat{\tau}^{2} \mathcal{R}_{t h}-$ $\lambda \nu \hat{\tau}^{3} / \log (2)$.

Let $\Delta \triangleq b^{2}-4 a c$. If $\Delta<0, x_{0}$ does not exist. Otherwise, (43) has two solutions $x_{1,2} \triangleq \frac{-b \pm \sqrt{\Delta}}{2 a}$. If $x_{1}, x_{2} \notin(0, \hat{\tau})$, $x_{0}$ does not exist. In the case when $x_{0}$ does not exist, the optimization problem is infeasible. However, in the case when $x_{0}$ exists, it is worth noting that there are two cases may occur here: 


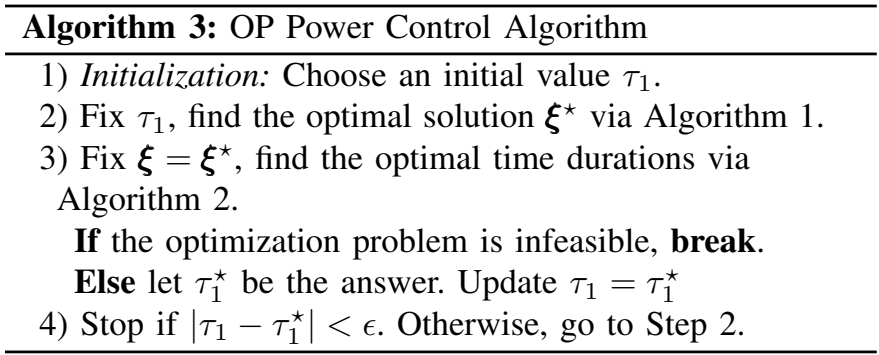

- Case 1: $f\left(x_{0}\right)<0$, which means that the answers for $f(x)=0$ does not exist. The optimization problem becomes infeasibility. Furthermore, it indicates that there may exist a hub with a spectral efficiency lower than $\mathcal{R}_{t h}$. This case occurs when the spectral efficiency threshold $\mathcal{R}_{t h}$ is set too high whereas this hub is experiencing unfavourable channel fading conditions or the distance from this hub to all APs are too far.

- Case 2: $f\left(x_{0}\right) \geq 0$, which implies that solutions for $f(x)=0$ exist. The optimal solution for $\tau_{1}$ is the maximum value attainable for $f(x)=0$ in range $\left(x_{0}, \hat{\tau}\right)$, which can be obtained using the bisection method, which is described in Algorithm 2.

3) Outage Prioritized Power Control Algorithm (OP Algorithm): By combining Algorithm 1 and 2, (35) can be solved using an iterative algorithm, which is summarized in Algorithm 3.

Complexity Analysis: Algorithm 1 solves the optimization problem (39) $N_{\text {loop }}$ times. Since (39) is a least square optimization problem and $\overline{\mathbf{G}} \in \mathbf{C}^{K \times\left(K N_{0}\right)}, \boldsymbol{\xi} \in \mathbf{C}^{K N_{0}}$, the complexity for solving (39) is $\mathcal{O}\left(K^{3} N_{0}^{2}\right)$. Therefore, the complexity of solving Algorithm 1 is $\mathcal{O}\left(N_{\text {loop }} K^{3} N_{0}^{2}\right)$. The per-iteration complexity for solving Algorithm 2 is $\mathcal{O}(1)$. Thus, the per-iteration complexity for solving Algorithm 3 is $\mathcal{O}\left(N_{\text {loop }} K^{3} N_{0}^{2}\right)$.

\section{Solving Optimization Problem Designed for SEP Mode}

1) Optimal Energy Allocation Weights: In the case when $\tau_{1}, \tau_{2}$ are fixed, the solution for the optimal power weights can be obtained by following Algorithm 1 .

2) Optimal Time Durations: Optimal time durations for the optimization problem (36) are proposed for the case when $\xi$ is fixed. By following a similar procedure to that for (40), our objective function (36a) becomes

$$
\min _{k} \tilde{\mathcal{R}}_{k}=\tau_{2} \log _{2}\left(1+\frac{\lambda \tau_{1}}{\mu \tau_{1}+\nu \tau_{2}}\right) .
$$

To solve the optimization problem (36) in the case when $\xi$ is fixed, we now need to deal with the following problem

$$
\begin{aligned}
\max _{\tau_{1}, \tau_{2}} & \tau_{2} \log _{2}\left(1+\frac{\lambda \tau_{1}}{\mu \tau_{1}+\nu \tau_{2}}\right) \\
\text { s.t } & \tau_{1}+\tau_{2}=\hat{\tau} \\
& \tilde{\mathcal{P}}_{k^{\prime}}\left(\tau_{1}, \tau_{2}\right) \leq \mathcal{P}_{t h} \\
& \tau_{1}, \tau_{2}>0 .
\end{aligned}
$$

From (33), the outage probability for the signal generated by hub $k^{\prime}$ can be rewritten as

$$
\tilde{\mathcal{P}}_{k^{\prime}}\left(\tau_{1}, \tau_{2}\right)= \begin{cases}1, & \text { if } \frac{\gamma_{t h}}{p_{k^{\prime}}} \geq r_{k^{\prime}} \\ 1-\Gamma\left[A_{k^{\prime}}, B_{k^{\prime}}\left(\frac{p_{k^{\prime}}}{\gamma_{t h}} r_{k^{\prime}}^{2}-r_{k^{\prime}}\right)\right], & \text { otherwise. }\end{cases}
$$

It is very important to note that since $\mathbf{p}=\frac{\tau_{1}}{\tau_{2}} \mathbf{G} \boldsymbol{\xi}$, the term (29) is proportional to $\frac{\tau_{1}}{\tau_{2}}$ whereas the term (30) is proportional to $\frac{\tau_{1}^{2}}{\tau_{2}^{2}}$. As a consequence, it is clear from (28) that the shape parameter $A_{k^{\prime}}$ does not contain $\tau_{1}$ and $\tau_{2}$, while the rate parameter $B_{k^{\prime}}$ is proportional to $\frac{\tau_{2}}{\tau_{1}}$. Following from this, it is clear that $B_{k^{\prime}} \frac{p_{k^{\prime}}}{\gamma_{t h}} r_{k^{\prime}}^{2}$ does not contain $\tau_{1}, \tau_{2}$. Therefore, in the case when $\xi$ is fixed, $A_{k^{\prime}}$ is a constant and we can denote

$$
B_{k^{\prime}} \frac{p_{k^{\prime}}}{\gamma_{t h}} r_{k^{\prime}}^{2} \triangleq w, \quad B_{k^{\prime}} r_{k^{\prime}} \triangleq \frac{\tau_{2}}{\tau_{1}} \times s,
$$

where $w, s$ are both constants. Thus, the outage probability for hub $k^{\prime}$ can be reduced to

$$
\tilde{\mathcal{P}}_{k^{\prime}}\left(\tau_{2}\right)= \begin{cases}1, & \text { if } \tau_{2} \geq \frac{\hat{\tau}}{1+\frac{\gamma t h}{\nu \mathbf{G}\left[k^{\prime}\right] \boldsymbol{\xi}}} \\ 1-\Gamma\left[A_{k^{\prime}}, w-\frac{s \tau_{2}}{\hat{\tau}-\tau_{2}}\right], & \text { otherwise. }\end{cases}
$$

Obviously, considering $\tau_{2}$ as a variable, this is an increasing function with $\tau_{2} \in[0, \hat{\tau})$. The minimum value of $\mathcal{P}\left(\tau_{2}\right)$ is equal to $\mathcal{P}(0)=1-\Gamma\left(A_{k^{\prime}}, w\right)$. It can be seen that if $1-\Gamma\left(A_{k^{\prime}}, w\right)>\mathcal{P}_{t h}$, the outage probability constraint (45c) will never be satisfied, which means that the optimization problem (45) is infeasible. However, if $1-\Gamma\left(A_{k^{\prime}}, w\right) \leq \mathcal{P}_{t h}$, by following the bisection method, it is possible to find $\tau^{\star}$ which satisfies $\mathcal{P}\left(\tau^{\star}\right)=\mathcal{P}_{t h}$. Thus, the outage probability constraint (45c) now is equivalent to $\tau_{2} \leq \tau^{\star}$. Now, for the sake of tractability, we denote $y=\tau_{2}$ and then, the optimization problem (45) can be reduced to

$$
\begin{array}{cl}
\max _{y} & y \log _{2}\left(1+\frac{\lambda y-\lambda \hat{\tau}}{(\mu-\nu) y-\mu \hat{\tau}}\right) \\
\text { s.t } & 0<y \leq \tau^{\star} .
\end{array}
$$

Let's define the objective function as

$$
g(y)=y \log _{2}\left(1+\frac{\lambda y-\lambda \hat{\tau}}{(\mu-\nu) y-\mu \hat{\tau}}\right) \text {, with } y \in(0, \hat{\tau}) .
$$

It is not difficult to show that $g^{\prime \prime}(y)<0$ with $\forall y \in(0, \hat{\tau})$. Therefore, $g(y)$ is a concave function in this domain, which indicates that the maximum value of $g(y)$ exists. The optimal value of $y$ can be found via solving the equation $g^{\prime}(y)=0$. The derivation of $g^{\prime}(y)$ is represented as

$$
\begin{aligned}
& g^{\prime}(y)=\log _{2}\left(1+\frac{\lambda y-\lambda \hat{\tau}}{(\mu-\nu) y-\mu \hat{\tau}}\right) \\
& -\frac{\log _{2}(e) \lambda \nu y}{((\mu-\nu) y-\mu \hat{\tau})((\lambda+\mu-\nu) y-\lambda \hat{\tau}-\mu \hat{\tau})} .
\end{aligned}
$$

It is worth noting that $\lim _{y \rightarrow 0} g^{\prime}(y)=\log _{2}\left(1+\frac{\lambda}{\mu}\right)>0$ and $\lim _{y \rightarrow \hat{\tau}} g^{\prime}(y)=-\frac{\lambda}{\log (2) \nu \hat{\tau}}<0$. As a result, there always exists a unique solution $y_{0}$ such that $g^{\prime}\left(y_{0}\right)=0$. This value can be found via the bisection method. It is worth noting that there are two cases which may occur here: 


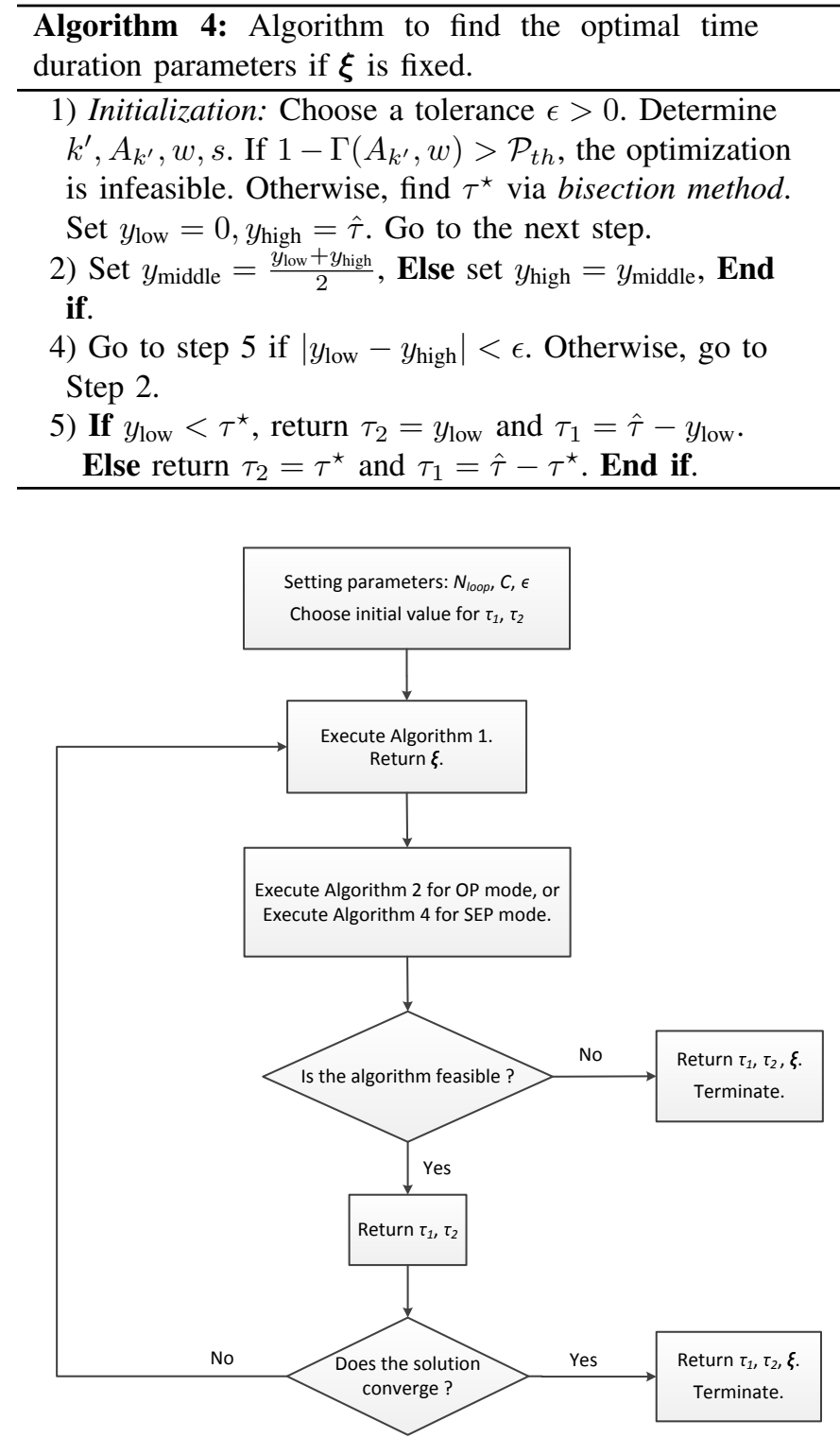

Fig. 4. Flowchart for the OP and SEP algorithm.

- If $y_{0} \leq \tau^{\star}$ : This means that the optimal value $y_{0}$ in the domain $(0, \hat{\tau})$ satisfies $(49 \mathrm{~b})$. Therefore, the optimal solution of (49) is $y_{0}$.

- If $y_{0}>\tau^{\star}$ : This means that the optimal value $y_{0}$ in the domain $(0, \hat{\tau})$ does not meet the condition (49b). Therefore, the optimal solution of (49) is $\tau^{\star}$.

The algorithm used to find out the time durations is summarized in Algorithm 4.

3) Spectral Efficiency Prioritized Power Control Algorithm (SEP Algorithm): We are able to solve the optimization (36) by iteratively computing Algorithm 1 and 4. This process is similar to that proposed in Algorithm 3, which is not repeated here for brevity. The complexity for computing the SEP algorithm is similar to that for solving Algorithm 3, which is $\mathcal{O}\left(N_{\text {loop }} K^{3} N_{0}^{2}\right)$.

The flowchart shown in Figure 4 illustrates the process of the proposed algorithms.
TABLE I

SYSTEM PARAMETERS USED FOR THE SIMULATIONS

\begin{tabular}{l|l}
\hline Parameters & Value \\
\hline Frequency $(f)$, bandwidth $(\mathrm{B})$ & $2.45 \mathrm{GHz}, 10.0 \mathrm{MHz}$ \\
Maximum transmit power $\left(P_{\mathrm{m}}\right)$ & $+30 \mathrm{dBm}[12]$ \\
Energy conversion efficiency $(\delta)$ & $0.35[24]$ \\
Time period for the first phase $\left(\tau_{0}\right)$ & 0 \\
Spectral efficiency threshold $\left(\mathcal{R}_{t h}\right)$ & $1.0 \mathrm{bit} / \mathrm{s} / \mathrm{Hz}$ \\
$\mathcal{P}_{t h}, \gamma_{t h}$ & $10 \%, 0 \mathrm{~dB}$ \\
Other path loss related parameters & $n_{1}=2.51, n_{2}=4.25, b_{1}=0.12$ \\
& $b_{2}=0.04, f_{0}=24.1 \mathrm{GHz}$ \\
& $D_{B P}=7.8 \mathrm{~m}, \sigma_{\mathrm{sh}}=7.65 \mathrm{~dB}$. \\
\hline
\end{tabular}

\section{AP Selection}

For a given user, there are APs located far away. Obviously, these APs do not contribute significantly to the harvested energy. This means that it is not necessary for all APs to participate in serving this user. In this paper, we adopt the Largest-Large-Scale-Fading-Based Selection scheme proposed in [33], which is effective and has low-complexity. In particular, $N_{0}$ APs with the largest average received useful signal powers are selected by each hub. For example, for hub $k, N_{0}$ APs whose the $N_{0}$ maximum $\beta_{n k}$ values are chosen.

\section{Numerical Results AND Discussions}

\section{A. Large-Scale Fading Model and Rician $\mathcal{K}$ Factor}

The large-scale fading coefficient $\beta_{n k}$ contains the path-loss and shadow fading, according to

$$
\beta_{n k}=\mathrm{PL}_{n k} \cdot 10^{\frac{\sigma_{\mathrm{sh}} z_{n k}}{10}},
$$

where $\mathrm{PL}_{n k}$, given by (53), represents the path-loss while $10^{\frac{\sigma_{\mathrm{sh}} z_{n k}}{10}}$ stands for the shadow fading with the standard deviation $\sigma_{\mathrm{sh}}$, and $z_{n k} \sim \mathcal{N}(0,1)$.

1) Path Loss Model and Shadowing: We use a dual-slope model proposed by the multi-frequency close-in (CI) model with a frequency-weighted path loss exponent (CIF) [34] for the path loss in an indoor environment. This model is described at the top of this page, where $\operatorname{FSPL}(f)$ is the free space path loss at $1 \mathrm{~m}$, given by $\operatorname{FSPL}(f)=20 \log \left(\frac{4 \pi f}{c}\right)$.

2) Rician $\mathcal{K}$ Factor: The Rician $\mathcal{K}$-factor and large-scale fading coefficients vary depending on the locations of hubs and APs. To model this phenomenon, we use the following formulation as in [35]:

$$
\mathcal{K}_{n k}=\frac{P_{\mathrm{LOS}}\left(D_{n k}\right)}{1-P_{\mathrm{LOS}}\left(D_{n k}\right)},
$$

where $D_{n k}$ (in meters) is the length of the considered link whereas $P_{\text {LOS }}$ is defined as in [34]:

$P_{\mathrm{LOS}}\left(D_{n k}\right)= \begin{cases}1, & \text { if } D_{n k} \leq 1 \mathrm{~m} \\ \exp \left(-\frac{D_{n k}-1}{4.9}\right), & \text { if } 1 \mathrm{~m}<D_{n k}<9.8 \mathrm{~m} \\ 0.17, & \text { if } D_{n k} \geq 9.8 \mathrm{~m} .\end{cases}$

\section{B. Parameters and Setup}

In all of the considered examples, we choose the parameters summarized in Table I. The transmit power is normalized by dividing the transmit power value (in $\mathrm{W}$ ) by the noise power, 


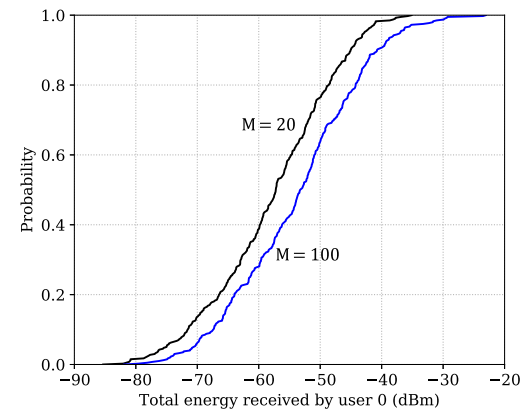

(a)

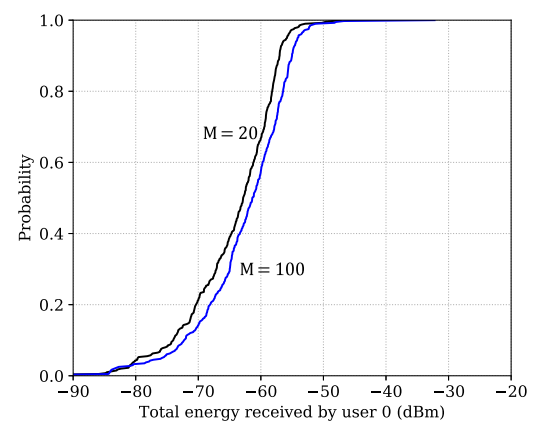

(b)

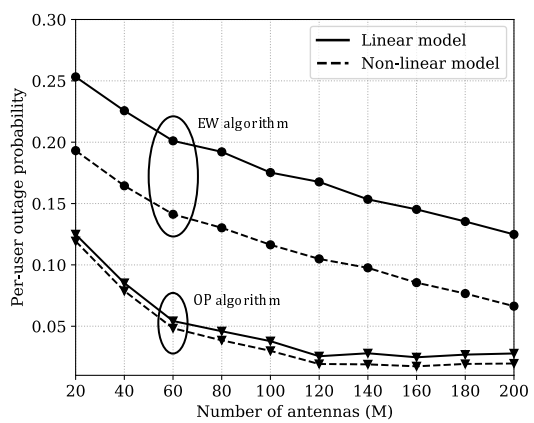

(c)

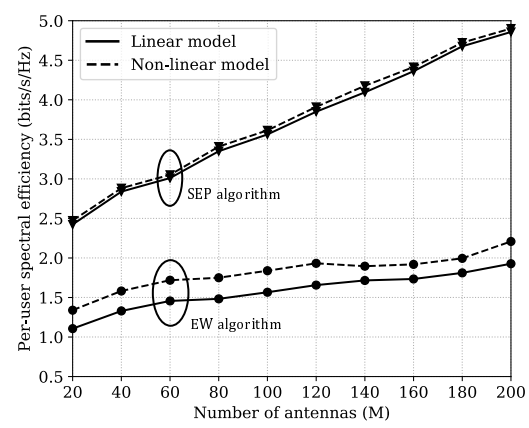

(d)

Fig. 5. In these simulations: $N=4, K=4, N_{0}=2$. (a) The total energy received at hub 0 under the $\mathrm{OP}$ algorithm; (b) The total energy received at hub 0 under the SEP algorithm; (c) The per-user outage probability for the OP algorithm by using the linear and non-linear model; (d) The per-user spectral efficiency for the SEP algorithm by using the linear and non-linear model.

where the noise power is defined in [21]. The deterministic vector $\mathbf{a}_{n k}$ whose components are given by

$$
\left[\mathbf{a}_{n k}\right]_{m}=e^{-j(m-1)(2 \pi d / \lambda) \sin \left(\phi_{n k}\right)}, \text { for } m=1, \cdots, M
$$

where $d$ is the antenna spacing, $\lambda$ is the wave length and $\phi_{n k}$ is the arrival angle which is distributed uniformly between 0 and $2 \pi$. In this simulation, we set $d=\lambda / 2$.

For each realization generated by the simulation, all users are uniformly distributed at random within a square area of $50 \mathrm{~m} \times 50 \mathrm{~m}$, whereas the location of APs remain unchanged. In particular, considering the deployed area as a chess board, each AP is located at the center of each square ${ }^{5}$. There were 500 realizations of the user locations and shadowing profiles generated during the evaluation. As a benchmark, we consider an equal weight $(\mathrm{EW})$ strategy where AP $n(\forall n=1, \cdots, N)$ designs power weights as

$$
\xi_{n, i}=\frac{1}{\text { the number of hubs in } \mathcal{H}_{n}}, \forall i \in \mathcal{H}_{n} .
$$

The time durations used by EW strategy are $\tau_{1}=0.8, \tau_{2}=$ 0.2. To allow a fair comparison between these mechanisms, the parameters in Table I and the AP selection method expressed in Section IV-D were used for all of the power control schemes. In addition, for each realization, the EW strategy was computed first to determine the minimum spectral efficiency (for OP mode) and the maximum outage probability (for SEP mode) among all the users. Subsequently, these values were used as the constraints for computing OP and SEP algorithm. This is to ensure that the proposed schemes and the EW power control always have an identical constraint.

\section{Results and Discussions}

1) Total Energy Received by Each Hub: We investigate the impact of a non-linear energy harvesting model on the system performance. This is crucial for validating the realistic utility of the proposed algorithms since the research conducted in [24] indicated that RF harvesting circuits typically have limiting sensitivities of between -85 and $-35 \mathrm{dBm}$. Figure 5(a) shows the CDF for the total energy received by hub 0 for the case when the OP algorithm is used. In this simulation, $N=4, K=4, N_{0}=2$ and there were 400 trials in total. For each trial, the location of the users was generated randomly within the area. As we can see from Figure 5(a), the more antennas are utilized by, the greater amount of energy user 0 receives. In addition, the result indicates that by using distributed massive MIMO combined with the OP algorithm, each hub is able to receive a reasonable amount of energy for use. As can be seen, the probability that the total amount of energy received by this user is lower than $-85 \mathrm{dBm}$ or larger than $-35 \mathrm{dBm}$ is very low for the both values of $M$. A similar result can be observed from Figure 5(b).

In Figure 5(c) and 5(d), we compare the system performance achieved under the linear energy harvesting model and the CLC model [24]. As can be seen, for the OP and SEP algorithm, the difference between the performance achieved by using the linear and CLC energy harvesting models is small. This is because the total amount of energy harvested by each user is normally between $-85 \mathrm{dBm}$ and $-35 \mathrm{dBm}$.

\footnotetext{
${ }^{5}$ This is to guarantee that all APs are evenly distributed in the region. As a result, in the simulation section, we only consider $N$ as a square number (e.g. $1,4,9, \cdots)$. This is only for the purposes of evaluation and does not affect the general insight.
} 


$$
\mathrm{PL}_{n k}=\left\{\begin{array}{l}
\operatorname{FSPL}(f)+10 n_{1}\left(1+b_{1} \frac{f-f_{0}}{f_{0}}\right) \log \left(\frac{D_{n k}}{1 \mathrm{~m}}\right), \quad \text { if } 1 \mathrm{~m}<D_{n k} \leq D_{B P} . \\
\operatorname{FSPL}(f)+10 n_{1}\left(1+b_{1} \frac{f-f_{0}}{f_{0}}\right) \log \left(\frac{D_{B P}}{1 \mathrm{~m}}\right)+10 n_{2}\left(1+b_{2} \frac{f-f_{0}}{f_{0}}\right) \log \left(\frac{D_{n k}}{D_{B P}}\right), \quad \text { if } D_{n k}>D_{B P} .
\end{array}\right.
$$

In comparison, for the EW strategy, the difference between the achieved performance under the linear and CLC model is found to be more significant. These results indicate that the proposed algorithms lessen and reduce the effect of limited harvesting sensitivity on the performance analysis.

2) OP Algorithm: We compare the outage probability performance of the OP algorithm with that of the EW strategy. Figure 6(a) represents the per-user outage probability with a different number of antennas under the EW scheme and the OP strategy with $N_{0} \in\{1,2\}$. In this simulation, $N=4$ and $K=4$. It can be clearly seen that the OP strategy outperforms the EW scheme for both values of $N_{0}$. For example, in the case when a low number of antennas are utilized at each AP $(M=20)$, about $28 \%$ of the received signals are in outage under the EW strategy, which is significantly higher than that under the OP algorithm with $N_{0}=1$ (under $20 \%$ ). The acquired per-user outage probability, however, is enhanced substantially when more antennas are added into each AP. This is because not only can utilizing more antennas increase the amount of wireless energy delivered toward each hub, but it is also capable of detecting uplink signals more effectively even when each hub transmits with a relatively low power. For example, when $M=200, N_{0}=1$, a per-user outage probability of approximately $13 \%$ and $4 \%$ is obtained for the EW and the OP strategies, respectively. The outage performance is improved even further when each user chooses more APs (e.g. $N_{0}=2$ ) with about $12 \%$ and $2 \%$ of received signals in outage under EW and OP power control, respectively. This result implies that the EW scheme is unable to meet the requirement of the IEEE 802.15.6 standard suggesting that the achieved outage probability should be lower than $10 \%$. Figure 6(b) shows the outage probability achieved under the OP scheme for different values of $\mathcal{R}_{t h}$. As can be seen, increasing the spectral efficiency threshold leads to an increase in outage probability. Both Figures 6(a) and 6(b) show that the proposed approximation performed well for a wide range of values of $M$ and $\mathcal{R}_{t h}$. Next, we investigate how $N_{0}$ affects the per-user outage probability. Figure 7(a) demonstrates the per-user outage probability obtained for 4 different values of $N_{0}$. In this simulation, $N=4, K=4$. In general, by allowing more APs to cooperate with each user, the outage probability performance improves. For example, compared to the case of choosing only one AP, by allowing 2 APs to assist each user, the per-user outage probability is enhanced significantly, by approximately $7 \%$ and $3 \%$ when $M=20$ and $M=200$, respectively. Interestingly, the system performance improves further when $N_{0}$ is increased from 2 to 4 , but not as significantly. This is because choosing more APs means that the probability that each user will experience a favorable channel increases. Nevertheless, in the context of wearable networks, not all the APs experience a favorable channel condition to each hub due to human-body blockage

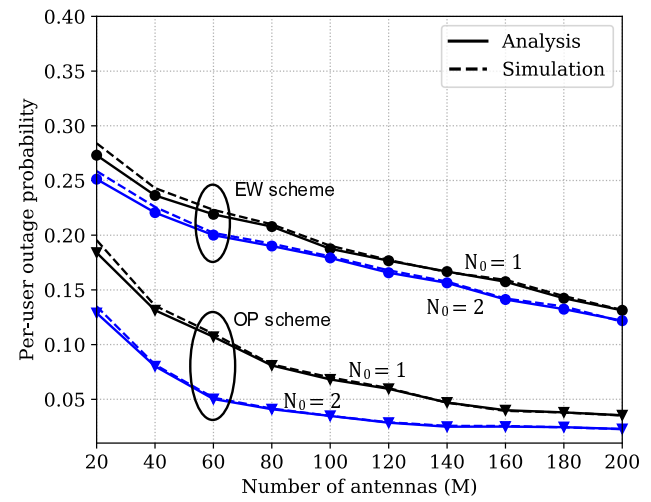

(a)

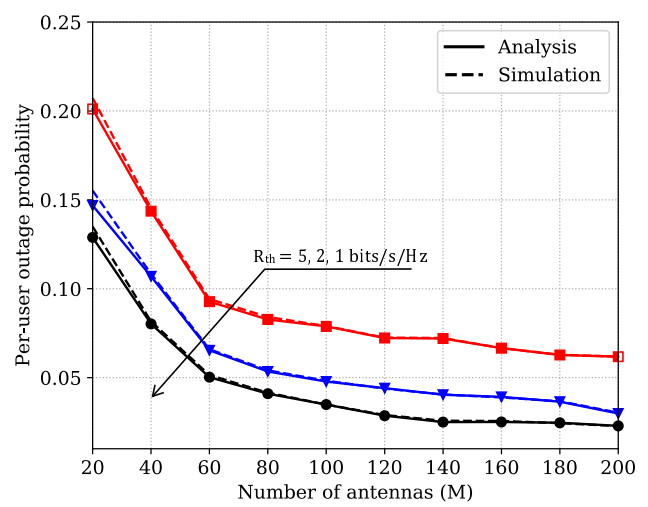

(b)

Fig. 6. (a) Per-user outage probability with various numbers of antennas under the EW and OP algorithms. Here, $N=4$ and $K=4$ and $N_{0}=2$; (b) Per-user outage probability with various values of $\mathcal{R}_{t h}$. In this simulation, $N=4, K=4$ and $N_{0}=2$.

phenomenon. As a result, in the case when $N_{0}$ is too large, each hub still accumulates energy from some APs which may not have favorable a channel condition (e.g. the APs which are too far away from the hub). It is worth noting that the IEEE standard can be satisfied for certain values of $M$. For example, by utilizing 40 antennas at each AP, and allowing each user to choose 2 APs, the per-user outage probability achieved under the OP algorithm is just $8 \%$.

Next we investigate three different scenarios to study the impact of varying $N$ and consequently $M$ on the system performance: Scenario 1 - Collocated setting with $N=$ $1, N_{0}=1, M=180 ;$ Scenario 2 - Distributed setting with $N=4, N_{0}=3, M=45$ and Scenario 3 - Distributed setting with $N=9, N_{0}=3, M=20$. Note that the total number of antennas utilized for each scenario are identical, which is 


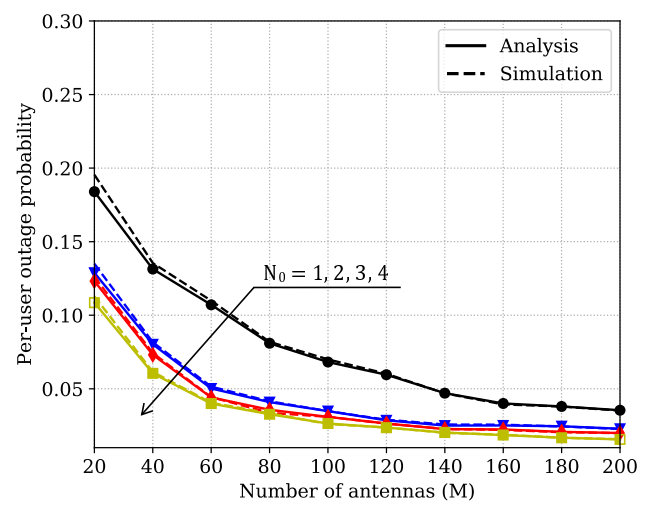

(a)

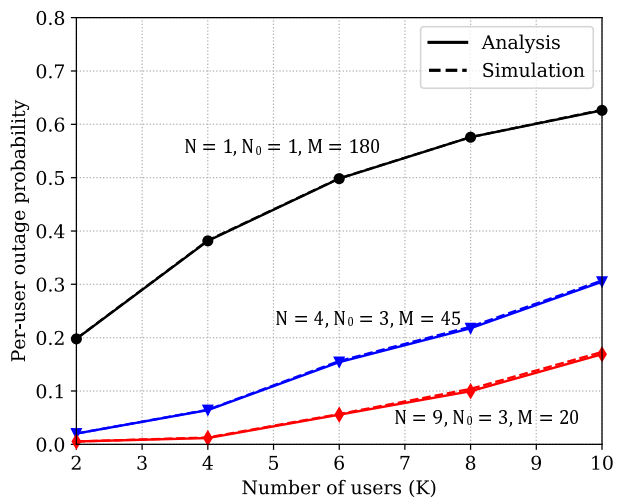

(b)

Fig. 7. (a) Per-user outage probability with 4 different values of $N_{0}$ under the OP algorithm. In this simulation, $N=4$ and $K=4$; (b) Per-user outage probability with different number of users under the OP algorithm for 3 scenarios: 1) $N=1, N_{0}=1, M=180$;2) $N=4, N_{0}=3, M=45$ and 3) $N=9, N_{0}=3, M=20$.

equal to 180 antennas in total. Figure 7(b) shows the peruser outage probability with different numbers of users for the 3 scenarios. As can be seen, Scenario 1 experiences the highest per-user outage probability with approximately $20 \%$ of signals in outage for the case $K=2$. This implies that wireless power transfer enabled by collocated massive MIMO may not provide an efficient performance in a wide area. This is a result of using only one AP which is unable to provide good coverage for all users. In comparison, using 4 APs each equipped with 45 antennas, Scenario 2 witnesses a significant performance improvement compared to Scenario 1. However, among the 3 scenarios, Scenario 3 exhibits the lowest per-user outage probability, about $1 \%$ and $6 \%$ for the case when $K=4$ and $K=6$, respectively. Scenario 3 uses up to 9 APs, which obviously provides better coverage compared to Scenario 1 and 2. An interesting point is that in Scenario 3, each user chooses only 3 APs, which means that each user uses only 60 antennas in total whereas in Scenario 2, each user uses up to 135 antennas. These results provide evidence for the efficiency of using distributed massive MIMO in this context.

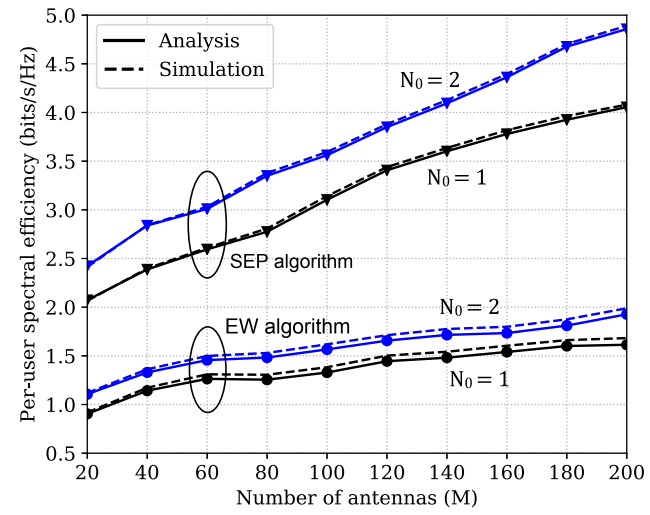

Fig. 8. Per-user spectral efficiency versus the number of antennas under the EW and SEP algorithms. Here, $N=4$ and $K=4$.

3) SEP Algorithm: Figure 8 compares the per-user spectral efficiency achieved under the EW and SEP schemes. As can be seen, the SEP scheme outperforms the EW algorithm significantly. Specifically, in the case when $M$ is low $(M=20)$ and $N_{0}=1$, each user achieves an average spectral efficiency typically less than 1 bits/s/Hz when using EW power control, which is approximately 1 bit lower than that achieved when using the SEP scheme. In comparison, in the case when $M$ is large $(M=200)$ and $N_{0}=1$, using the SEP scheme is able to achieve a per-user spectral efficiency of approximately 4.0 bits $/ \mathrm{s} / \mathrm{Hz}$, which is nearly double that for the EW algorithm (around $1.7 \mathrm{bits} / \mathrm{s} / \mathrm{Hz}$ ).

Figure 9(a) shows the per-user spectral efficiency under the SEP scheme for 4 values of $N_{0}$, i.e. $N_{0} \in\{1,2,3,4\}$. Here, $N=4, K=4$. Similar to the insight obtained from Figure 7(a), it can be seen that allowing each user to choose more APs leads to an improved spectral efficiency performance. However, it is worth emphasizing that the choosing more than 2 APs may not result in a significant performance improvement. For example, considering $M=140$, utilizing the SEP algorithm achieves approximately 3.6, 4.1 and 4.2 bits/s/Hz for the case when $N_{0}=1,2$ and 3 , respectively. Figure 9(b) represents the per-user spectral efficiency achieved for the three scenarios with various values of $K$. The figure reveals that by distributing more APs over an area, the spectral efficiency performance can be enhanced. Regarding the distributed setting, taking $K=4$ as an example, each user is able to achieve an average spectral efficiency of $4.0 \mathrm{bits} / \mathrm{s} / \mathrm{Hz}$ for the case when 9 APs (each with 20 antennas) are spread out over the area (Scenario 3), which is 1.5 bits higher than that for the case when only 4 APs (each with 45 antennas) are utilized (Scenario 2). In comparison, when it comes to the collocated setting where only one AP with a very large number of antennas $(M=180)$ is used (Scenario 1$)$, the achieved peruser spectral efficiency is much lower (just about $1.1 \mathrm{bits} / \mathrm{s} / \mathrm{Hz}$ for the all considered $K$ values). This result again emphasizes the potential of distributed massive MIMO in the context of wearable applications.

4) Convergence Analysis: It is important to note that using the iterative method may not achieve the global-optimal solution in all cases. However, as shown in the numerical results, the proposed algorithms still improve the network performance 


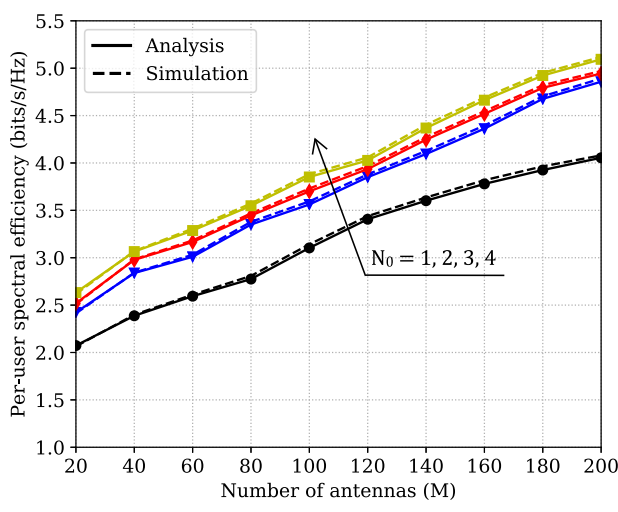

(a)

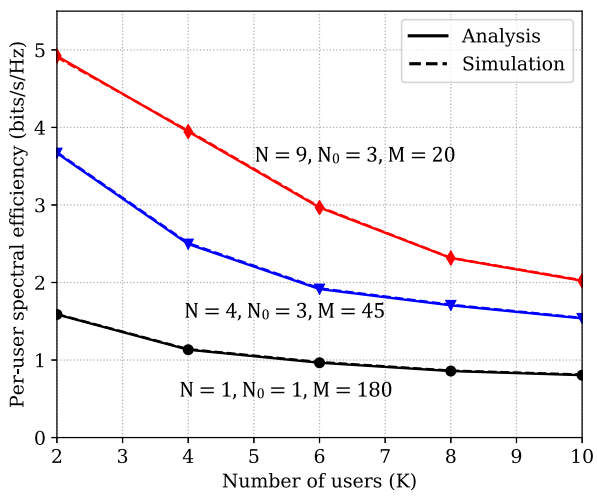

(b)

Fig. 9. (a) Per-user spectral efficiency with 4 different values of $N_{0}$ under the SEP algorithm. In this simulation, $N=4$ and $K=4$; (b) Per-user spectral efficiency with different number of users under the SEP algorithm for 3 scenarios: 1) $N=1, N_{0}=1, M=180$; 2) $N=4, N_{0}=3, M=45$ and 3) $N=9, N_{0}=3, M=20$.

significantly. We now evaluate the convergence behavior of the proposed schemes. For a number of iterations, Figure 10(a) shows the maximum outage probability obtained via the OP algorithm while Figure 10(b) illustrates the achieved minimum spectral efficiency via the SEP scheme. Our numerical results show that the both methods converge very fast, within about 8 iterations. As expected, the convergence speed tends to increase with more users added into the network.

\section{CONCLUSIONS}

We investigated the use of distributed massive MIMO to power and serve the data requirements of wearable devices. We considered a system model in which there are multiple APs and multiple users while the fading is Rician distributed. In addition, we have introduced a tight approximation for the outage probability and the spectral efficiency, which are tractable and well suited for use in further analyses and system designs. Based on this, we then developed 2 power control mechanisms which are specifically designed for two different operation modes namely OP and SEP algorithms. The results showed that while the OP algorithm satisfies the

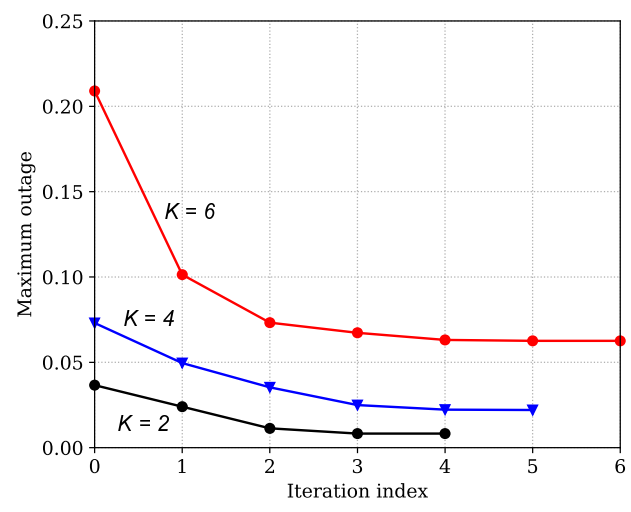

(a)

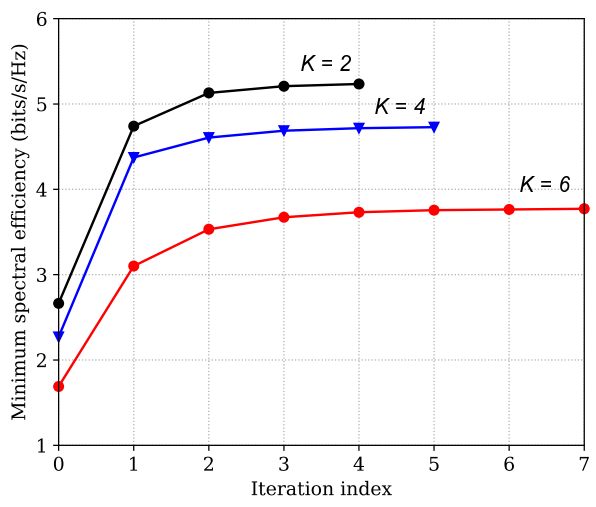

(b)

Fig. 10. (a) The maximum outage probability versus the number of iterations, OP algorithm. Here, $N=9, M=40 N_{0}=2$; (b) The minimum spectral efficiency versus the number of iterations, SEP algorithm. Here, $N=9, M=$ $80 N_{0}=2$.

IEEE 802.15.6 standard with a certain number of AP antennas, the SEP scheme is able to provide each user with an average spectral efficiency of more than $1 \mathrm{bits} / \mathrm{s} / \mathrm{Hz}$.

This study has only focused on wearable networks powered by massive MIMO where it was assumed that the APs have perfect CSI. Nevertheless, in practice, the CSI needs to be estimated at the AP in the uplink phase. As a result, an important research direction is to consider channel estimation in future. Additionally, the use of more advanced decoders, such as those employing joint ZF/MMSE or optimal precoder, at the APs may improve the system performance further.

\section{APPENDIX}

\section{A. Proof of Lemma 2}

The formula (12) can be easily proved by using the definition of the vector $\mathbf{l}_{k k}$ and noting that $\mathbb{E}\left[\mathbf{h}_{n k}\right]^{2}=M \beta_{n k}$ [27]

$$
\mathbb{E}\left\{\left\|\mathbf{l}_{k k}\right\|^{2}\right\}=\mathbb{E}\left\{\sum_{n \in \mathcal{A}_{k}}\left\|\mathbf{h}_{n k}\right\|^{2}\right\}=M \sum_{n \in \mathcal{A}_{k}} \beta_{n k} .
$$




\section{B. Proof of Lemma 3}

Regarding Lemma 3 for the case $i=k$, we have the following transformations

$$
\begin{aligned}
\mathbb{E}\left\{\left\|\mathbf{l}_{k k}\right\|^{4}\right\} & =\mathbb{E}\left\{\left\|\mathbf{h}_{k k}\right\|^{4}\right\} \\
& +\sum_{n \neq m \in \mathcal{A}_{k}} \mathbb{E}\left\{\left\|\mathbf{h}_{n k}\right\|^{2}\right\} \mathbb{E}\left\{\left\|\mathbf{h}_{m k}\right\|^{2}\right\} .
\end{aligned}
$$

By using 1 and $\mathbb{E}\left[\mathbf{h}_{n k}\right]^{2}=M \beta_{n k}$, the proof for this case is completed. The proof for the case $i \neq k$ can be executed similarly as follows.

$$
\begin{aligned}
\mathbb{E}\left\{\left|\mathbf{l}_{k k}^{H} \mathbf{l}_{k i}\right|^{2}\right\} & =\sum_{n \in \mathcal{A}_{k}} \mathbb{E}\left\{\left|\mathbf{h}_{n k}^{H} \mathbf{h}_{n i}\right|^{2}\right\} \\
& +\sum_{n \neq m \in \mathcal{A}_{k}} \mathbb{E}\left\{\mathbf{h}_{n i}^{H} \mathbf{h}_{n k} \mathbf{h}_{m k}^{H} \mathbf{h}_{m i}\right\} .
\end{aligned}
$$

Since $\mathbf{h}_{n i}, \mathbf{h}_{n k}, \mathbf{h}_{m i}, \mathbf{h}_{m k}$ are mutually independent with $n \neq$ $m$ and $i \neq k, \mathbb{E}\left\{\mathbf{h}_{n i}^{H} \mathbf{h}_{n k} \mathbf{h}_{m k}^{H} \mathbf{h}_{m i}\right\}=\overline{\mathbf{h}}_{n i}^{H} \overline{\mathbf{h}}_{n k} \overline{\mathbf{h}}_{m k}^{H} \overline{\mathbf{h}}_{m i}$. By substituting this into (60), Lemma 3 is proved.

\section{Proof of Lemma 4}

According to the law of large numbers, we have $\frac{1}{M}\left\|\mathbf{h}_{n k}\right\|^{2} \stackrel{\text { a.s. }}{\rightarrow} \beta_{n k}$ as $M \rightarrow \infty$. Subsequently, it can easily be used to provide a proof for (14) as follows

$$
\frac{1}{M}\left\|\mathbf{l}_{k k}\right\|^{2}=\sum_{n \in \mathcal{A}_{k}} \frac{\left\|\mathbf{h}_{n k}\right\|^{2}}{M} \stackrel{a . s .}{\rightarrow} \sum_{n \in \mathcal{A}_{k}} \beta_{n k}, \text { as } M \rightarrow \infty .
$$

\section{Proof of Lemma 6}

Substituting (2) into (3), the expected energy harvested by hub $k$ can be formulated as

$$
\mathbb{E}\left\{\left|y_{k}\right|^{2}\right\}=\mathbb{E}\left\{\left|\sum_{n=1}^{N} \sum_{i \in \mathcal{H}_{n}} \sqrt{\xi_{n, i} P_{\mathrm{m}}} \frac{\mathbf{h}_{n k}^{H} \mathbf{h}_{n i}}{\left\|\mathbf{h}_{n i}\right\|} x_{n i}\right|^{2}\right\},
$$

where the expectation is taken over $x_{n i}$. Since the signal $x_{n i}, x_{m i}$ and $x_{n j}$ are mutually independent with $n \neq m, i \neq j$, and $\left\{\left\|x_{n i}\right\|^{2}\right\}=1$, the above equation can be reduced to

$$
\begin{aligned}
& \mathbb{E}\left\{\left|y_{k}\right|^{2}\right\}=\sum_{n=1}^{N} \sum_{i \in \mathcal{H}_{n}} \xi_{n, i} P_{\mathrm{m}} \frac{\left|\mathbf{h}_{n k}^{H} \mathbf{h}_{n i}\right|^{2}}{\left\|\mathbf{h}_{n i}\right\|^{2}} \\
& =\sum_{n \in \mathcal{A}_{k}} \xi_{n, k} P_{\mathrm{m}}\left\|\mathbf{h}_{n k}\right\|^{2}+\sum_{n=1}^{N} \sum_{\substack{i \neq k \\
i \in \mathcal{H}_{n}}} \xi_{n, i} P_{\mathrm{m}} \frac{\left|\mathbf{h}_{n k}^{H} \mathbf{h}_{n i}\right|^{2}}{\left\|\mathbf{h}_{n i}\right\|^{2}} .
\end{aligned}
$$

Note that Lemma 4 holds true with every values of $n$. Using Lemma 4 with $\left|\mathcal{A}_{k}\right|=1$ and Lemma 1, the asymptotic energy harvested by hub $k$ can be derived as

$$
\mathbb{E}\left\{\left|y_{k}\right|^{2}\right\} \approx \sum_{n \in \mathcal{A}_{k}} M \beta_{n k} P_{\mathrm{m}} \xi_{n, k}+\sum_{n=1}^{N} \sum_{\substack{i \neq k \\ i \in \mathcal{H}_{n}}} \frac{g_{n}[k, i]}{M \beta_{n i}} P_{\mathrm{m}} \xi_{n, i}
$$

By substituting $(64,4)$ into $(5)$, Lemma 6 is proved.

\section{E. Proof of Proposition 1}

Formula (21) can be obtained by applying directly Lemmas 4,5 , and 6 on the formula (16). Regarding (22), by using the approximation $\mathbb{E}\left\{\frac{X}{Y}\right\} \approx \frac{\mathbb{E}\{X\}}{\mathbb{E}\{Y\}}$ when $M$ is large [27], we obtain

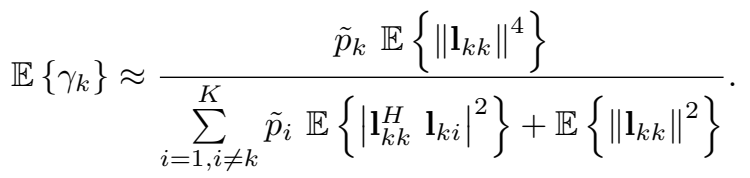

By using Lemmas 2 and 3, the proof is completed.

\section{F. Proof of Proposition 2}

When $M$ is sufficiently large, by using the approximation $\mathbb{E}\left\{\log _{2}\left(1+\frac{X}{Y}\right)\right\} \approx \log _{2}\left(1+\frac{\mathbb{E}\{X\}}{\mathbb{E}\{Y\}}\right)$ [27], we have

$$
\begin{aligned}
\mathcal{R}_{k} & \approx \tau_{2} \log _{2}\left(1+\mathbb{E}\left\{\gamma_{k}\left(\tau_{1}, \tau_{2}, \boldsymbol{\xi}\right)\right\}\right) \\
& =\tau_{2} \log _{2}\left(1+\frac{\tilde{p}_{k} q_{k k}}{\sum_{i=1, i \neq k}^{K} \tilde{p}_{i} q_{k i}+r_{k}}\right),
\end{aligned}
$$

where Proposition 1 was used in the last transformation.

\section{G. Proof of Lemma 7}

By utilizing Lemma 3, we have the following equations

$$
\begin{aligned}
y_{m} & =\mathbb{E}\left\{\mathbf{l}_{k k}^{H} \mathbf{l}_{k i}\right\}=\overline{\mathbf{l}}_{k k}^{H} \overline{\mathbf{l}}_{k i}, \\
y_{v} & =\mathbb{E}\left\{\left|\mathbf{l}_{k k}^{H} \mathbf{l}_{k i}\right|^{2}\right\}-\left|\mathbb{E}\left\{\mathbf{l}_{k k}^{H} \mathbf{l}_{k i}\right\}\right|^{2}=q_{k i}-\left|\overline{\mathbf{l}}_{k k}^{H} \overline{\mathbf{l}}_{k i}\right|^{2} .
\end{aligned}
$$

When $M$ is sufficiently large, according to the central limit theorem [36], the term $\mathbf{l}_{k k}^{H} \mathbf{l}_{k i}$ can be approximated as a complex Gaussian random variable $Y$ whose mean and variance are identical to that of $\mathbf{l}_{k k}^{H} \mathbf{l}_{k i}$. In other words, $\mathbf{l}_{k k}^{H} \mathbf{l}_{k i} \approx Y$, where $Y \sim \mathcal{C N}\left(y_{m}, y_{v}\right) . Y$ can be rewritten as $Y=Y_{r}+j Y_{i}$, where $Y_{r}$ and $Y_{i}$ represent the real and the imaginary part of $Y$, respectively. Note that $Y_{r}$ are independent from $Y_{i}$ and $Y_{r} \sim \mathcal{N}\left(\Re\left(y_{m}\right), \frac{y_{v}}{2}\right) ; Y_{i} \sim \mathcal{N}\left(\Im\left(y_{m}\right), \frac{y_{v}}{2}\right)$. As a result, when $M$ is sufficiently large, term 1 can be approximated as

$$
\begin{aligned}
\text { term } 1 & \approx \mathbb{E}\left\{|Y|^{4}\right\}=\mathbb{E}\left\{\left|Y_{r}+j Y_{i}\right|^{4}\right\} \\
& =\mathbb{E}\left\{Y_{r}^{4}\right\}+2 \mathbb{E}\left\{Y_{r}^{2} Y_{i}^{2}\right\}+\mathbb{E}\left\{Y_{i}^{4}\right\} .
\end{aligned}
$$

To compute this, it is important to remember that

$$
\begin{aligned}
& \mathbb{E}\left\{Y_{r}^{4}\right\}=\Re\left(y_{m}\right)^{4}+3 y_{v} \Re\left(y_{m}\right)^{2}+\frac{3 y_{v}^{2}}{4}, \\
& \mathbb{E}\left\{Y_{r}^{2}\right\}=\Re\left(y_{m}\right)^{2}+\frac{y_{v}}{2}, \\
& \mathbb{E}\left\{Y_{i}^{4}\right\}=\Im\left(y_{m}\right)^{4}+3 y_{v} \Im\left(y_{m}\right)^{2}+\frac{3 y_{v}^{2}}{4}, \\
& \mathbb{E}\left\{Y_{i}^{2}\right\}=\Im\left(y_{m}\right)^{2}+\frac{y_{v}}{2} .
\end{aligned}
$$

By plugging (69) into (68) and performing several mathematical manipulations, the proof of Lemma 7 is accomplished. 


\section{H. Proof of Lemma 8}

We start to investigate term 2 by writing it as

$$
\begin{aligned}
\text { term } 2 & =\mathbb{E}\left\{\left|\mathbf{l}_{k k}^{H} \mathbf{l}_{k i}\right|^{2}\left|\mathbf{l}_{k k}^{H} \mathbf{l}_{k j}\right|^{2}\right\} \\
& =\mathbb{E}\left\{\mathbf{l}_{k k}^{H} \mathbf{l}_{k i} \mathbf{l}_{k i}^{H} \mathbf{l}_{k k} \mathbf{l}_{k k}^{H} \mathbf{l}_{k j} \mathbf{l}_{k j}^{H} \mathbf{l}_{k k}\right\} .
\end{aligned}
$$

It is important to note that

$$
\begin{aligned}
\mathbb{E}\left\{\mathbf{l}_{k i} \mathbf{l}_{k i}^{H}\right\} & =\mathbf{L}_{k i}+\overline{\mathbf{l}}_{k i} \overline{\mathbf{l}}_{k i}^{H}=\mathbf{B}, \\
\mathbb{E}\left\{\mathbf{l}_{k j} \mathbf{l}_{k j}^{H}\right\} & =\mathbf{L}_{k j}+\overline{\mathbf{l}}_{k j} \overline{\mathbf{l}}_{k j}^{H}=\mathbf{C} .
\end{aligned}
$$

Since $\mathbf{l}_{k k}, \mathbf{l}_{k i}$ and $\mathbf{l}_{k j}$ are mutually independent, term 2 now can be rewritten as

$$
\begin{aligned}
& \text { term } 2=\mathbb{E}\left\{\mathbf{l}_{k k}^{H} \mathbf{B} \mathbf{l}_{k k} \mathbf{l}_{k k}^{H} \mathbf{C l}_{k k}\right\} \\
& \stackrel{(a)}{=} \operatorname{Tr}\left[\mathbf{B} \mathbf{L}_{k k}\left(\mathbf{C}+\mathbf{C}^{H}\right) \mathbf{L}_{k k}\right]+\overline{\mathbf{l}}_{k k}^{H}\left(\mathbf{B}+\mathbf{B}^{H}\right) \mathbf{L}_{k k}\left(\mathbf{C}+\mathbf{C}^{H}\right) \overline{\mathbf{l}}_{k k} \\
& +\left(\operatorname{Tr}\left(\mathbf{L}_{k k} \mathbf{B}\right)+\overline{\mathbf{l}}_{k k}^{H} \mathbf{B} \overline{\mathbf{l}}_{k k}\right)\left(\operatorname{Tr}\left(\mathbf{L}_{k k} \mathbf{C}\right)+\overline{\mathbf{l}}_{k k}^{H} \mathbf{C} \overline{\mathbf{l}}_{k k}\right),
\end{aligned}
$$

where the transformation (a) was implemented by using [37, 7.2.4]. Subsequently, using the Hermitian properties (e.g. $\mathbf{B}=\mathbf{B}^{H}$ and $\mathbf{C}=\mathbf{C}^{H}$ ) combined with several algebraic manipulations, the proof of Lemma 8 is completed.

\section{REFERENCES}

[1] S. Movassaghi, M. Abolhasan, J. Lipman, D. Smith, and A. Jamalipour, "Wireless body area networks: A survey," IEEE Commun. Surv. Tut., vol. 16, no. 3, pp. 1658-1686, Jan. 2014.

[2] S. Xiao, A. Dhamdhere, V. Sivaraman, and A. Burdett, "Transmission power control in body area sensor networks for healthcare monitoring," IEEE J. Sel. Areas Commun., vol. 27, no. 1, pp. 37-48, January 2009.

[3] M. Chen, S. Gonzalez, A. Vasilakos, H. Cao, and V. C. Leung, "Body area networks: A survey," Mobile networks and applications, vol. 16, no. 2, pp. 171-193, 2011.

[4] H. Sun, Z. Zhang, R. Q. Hu, and Y. Qian, "Wearable communications in 5G: challenges and enabling technologies," IEEE Veh. Technol. Mag., vol. 13, no. 3, pp. 100-109, Sept. 2018.

[5] S. Ulukus, A. Yener, E. Erkip, O. Simeone, M. Zorzi, P. Grover, and K. Huang, "Energy harvesting wireless communications: A review of recent advances," IEEE J. Sel. Areas Commun., vol. 33, no. 3, pp. 360381, Mar. 2015.

[6] L. Liu, R. Zhang, and K.-C. Chua, "Wireless information transfer with opportunistic energy harvesting," IEEE Trans. Wireless Commun., vol. 12, no. 1, pp. 288-300, Jan. 2013.

[7] A. A. Nasir, X. Zhou, S. Durrani, and R. A. Kennedy, "Wirelesspowered relays in cooperative communications: Time-switching relaying protocols and throughput analysis," IEEE Trans. Commun., vol. 63, no. 5, pp. 1607-1622, May 2015.

[8] S. Zhou, M. Zhao, X. Xu, J. Wang, and Y. Yao, "Distributed wireless communication system: a new architecture for future public wireless access," IEEE Commun. Mag., vol. 41, no. 3, pp. 108-113, Mar. 2003.

[9] X. Chen, X. Wang, and X. Chen, "Energy-efficient optimization for wireless information and power transfer in large-scale MIMO systems employing energy beamforming," IEEE Wireless Commun. Lett., vol. 2, no. 6, pp. 667-670, Dec. 2013.

[10] T. A. Khan, A. Yazdan, and R. W. Heath, "Optimization of power transfer efficiency and energy efficiency for wireless-powered systems with massive MIMO," IEEE Trans. Wireless Commun., vol. 17, no. 11, pp. 7159-7172, Nov. 2018.

[11] S. Kashyap, E. Björnson, and E. G. Larsson, "On the feasibility of wireless energy transfer using massive antenna arrays," IEEE Trans. Wireless Commun., vol. 15, no. 5, pp. 3466-3480, May 2016.

[12] G. Yang, C. K. Ho, R. Zhang, and Y. L. Guan, "Throughput optimization for massive MIMO systems powered by wireless energy transfer," IEEE J. Sel. Areas Commun., vol. 33, no. 8, pp. 1640-1650, Aug. 2015.

[13] C. Feng, Y. Jing, and S. Jin, "Interference and outage probability analysis for massive MIMO downlink with MF precoding," IEEE Signal Process. Lett., vol. 23, no. 3, pp. 366-370, Mar. 2016.
[14] S. Atapattu, P. Dharmawansa, C. Tellambura, and J. Evans, "Exact outage analysis of multiple-user downlink with MIMO matched-filter precoding," IEEE Commun. Lett., vol. 21, no. 12, pp. 2754-2757, Dec. 2017.

[15] D. Ciuonzo, P. S. Rossi, and S. Dey, "Massive MIMO channel-aware decision fusion," IEEE Trans. Signal Process., vol. 63, no. 3, pp. 604619, Feb. 2015.

[16] A. Shirazinia, S. Dey, D. Ciuonzo, and P. Salvo Rossi, "Massive MIMO for decentralized estimation of a correlated source," IEEE Trans. Signal Process., vol. 64, no. 10, pp. 2499-2512, May 2016.

[17] F. Jiang, J. Chen, A. L. Swindlehurst, and J. A. Lpez-Salcedo, "Massive MIMO for wireless sensing with a coherent multiple access channel," IEEE Trans. Signal Process., vol. 63, no. 12, pp. 3005-3017, June 2015.

[18] G. Dong, H. Zhang, and D. Yuan, "Downlink achievable rate of massive MIMO enabled SWIPT systems over rician channels," IEEE Commun. Lett., vol. 22, no. 3, pp. 578-581, Mar. 2018.

[19] G. Taricco and G. Coluccia, "Optimum receiver design for correlated rician fading MIMO channels with pilot-aided detection," IEEE J. Sel. Areas Commun., vol. 25, no. 7, pp. 1311-1321, Sept. 2007.

[20] S. L. Cotton and W. G. Scanlon, "A statistical analysis of indoor multipath fading for a narrowband wireless body area network," in 2006 IEEE 17th International Symposium on Personal, Indoor and Mobile Radio Communications, Sept. 2006, pp. 1-5.

[21] H. Q. Ngo, A. Ashikhmin, H. Yang, E. G. Larsson, and T. L. Marzetta, "Cell-free massive MIMO versus small cells," IEEE Trans. Wireless Commun., vol. 16, no. 3, pp. 1834-1850, Mar. 2017.

[22] Ruijun Fu et al., "Doppler spread analysis of human motions for Body Area Network applications," in 2011 IEEE 22nd International Symposium on Personal, Indoor and Mobile Radio Communications, Sep. 2011, pp. 2209-2213.

[23] D. Smith, L. Hanlen, J. Zhang, D. Miniutti, D. Rodda, and B. Gilbert, "Characterization of the dynamic narrowband on-body to off-body area channel," in 2009 IEEE International Conference on Communications, June 2009, pp. 1-6.

[24] P. N. Alevizos and A. Bletsas, "Sensitive and nonlinear far-field RF energy harvesting in wireless communications," IEEE Trans. Wireless Communs., vol. 17, no. 6, pp. 3670-3685, June 2018.

[25] H. Dai, Y. Liu, G. Chen, X. Wu, T. He, A. X. Liu, and H. Ma, "Safe charging for wireless power transfer," IEEE/ACM Transactions on Networking, vol. 25, no. 6, pp. 3531-3544, Dec. 2017.

[26] T. L. Marzetta, E. G. Larsson, H. Yang, and H. Q. Ngo, Fundamentals of massive MIMO. Cambridge University Press, 2016.

[27] Q. Zhang, S. Jin, K.-K. Wong, H. Zhu, and M. Matthaiou, "Power scaling of uplink massive MIMO systems with arbitrary-rank channel means," IEEE J. Sel. Topics Signal Process., vol. 8, no. 5, pp. 966-981, Oct. 2014.

[28] J. Hoydis, "Random matrix theory for advanced communication systems." 2012

[29] R. W. Heath, M. Kountouris, and T. Bai, "Modeling heterogeneous network interference using Poisson Point Processes," IEEE Trans. Signal Process., vol. 61, no. 16, pp. 4114-4126, Aug. 2013.

[30] I. S. Gradshteyn and I. M. Ryzhik, Table of integrals, series, and products. Academic press, 2014.

[31] H. V. Cheng, E. Bjrnson, and E. G. Larsson, "Optimal pilot and payload power control in single-cell massive MIMO systems," IEEE Trans. Signal Process., vol. 65, no. 9, pp. 2363-2378, May 2017.

[32] S. Diamond and S. Boyd, "CVXPY: A Python-embedded modeling language for convex optimization," Journal of Machine Learning Research, vol. 17 , no. 83, pp. 1-5, 2016.

[33] H. Q. Ngo, L. Tran, T. Q. Duong, M. Matthaiou, and E. G. Larsson, "On the total energy efficiency of cell-free massive MIMO," IEEE Trans. Green Commun. Netw., vol. 2, no. 1, pp. 25-39, Mar. 2018.

[34] K. Haneda et al., "Indoor 5G 3GPP-like channel models for office and shopping mall environments," in 2016 IEEE International Conference on Communications Workshops (ICC), May 2016, pp. 694-699.

[35] A. H. Jafari, D. Lopez-Perez, M. Ding, and J. Zhang, "Study on scheduling techniques for ultra dense small cell networks," in 2015 IEEE 82nd Vehicular Technology Conference (VTC2015-Fall), Sep. 2015, pp. $1-6$.

[36] R. D. Yates and D. J. Goodman, "Probability and stochastic processes," John Willey \& Sons, 1999.

[37] K. B. Petersen, M. S. Pedersen et al., "The matrix cookbook," Technical University of Denmark, vol. 7, no. 15, p. 510, 2008 